\title{
Indium-111 labelling of liposomal HEGF for radionuclide delivery via ultrasound-induced cavitation
}

Joshua Owen ${ }^{1}$, Eloise Thomas' ${ }^{2}$, Jyothi Menon ${ }^{2}$, Michael Gray ${ }^{1}$, Irini Skaripa-Koukelli², Martin R. Gill2, Sheena Wallington ${ }^{2}$, Rebecca L. Miller ${ }^{2}$, Katherine Vallis*2 and Robert Carlisle*1.

${ }^{1}$ Department of Engineering Science, Institute of Biomedical Engineering, University of Oxford, Old Road Campus Research Building, Oxford OX3 7DQ

${ }^{2}$ CRUK/MRC Oxford Institute for Radiation Oncology, Department of Oncology, University of Oxford, Old Road Campus, Oxford, OX3 7DQ

*Both authors contributed equally

Keywords: ultrasound, nanoparticles, radiotherapy, radiopharmaceuticals, liposomes, enhanced delivery, cavitation 
47

48

49

\section{Abstract}

The purpose of this exploratory study was to investigate the combination of a radiopharmaceutical, nanoparticles and ultrasound (US) enhanced delivery to develop a clinically viable therapeutic strategy for tumours overexpressing the epidermal growth factor receptor (EGFR). Molecularly targeted radionuclides have great potential for cancer therapy but are sometimes associated with insufficient delivery resulting in sub-cytotoxic amounts of radioactivity being delivered to the tumour. Liposome formulations are currently used in the clinic to reduce the side effects and improve the pharmacokinetic profile of chemotherapeutic drugs. However, in contrast to non-radioactive agents, loading and release of radiotherapeutics from liposomes can be challenging in the clinical setting. US-activated cavitation agents such as microbubbles (MBs) have been used to release therapeutics from liposomes to enhance the distribution/delivery in a target area. In an effort to harness the benefits of these techniques, the development of a liposome loaded radiopharmaceutical construct for enhanced delivery via acoustic cavitation was studied. The liposomal formulation was loaded with peptide, human epidermal growth factor (HEGF), coupled to a chelator for subsequent radiolabelling with ${ }^{111}$ Indium $\left(\left[{ }^{111} \ln \right] \ln { }^{3+}\right)$, in a manner designed to be compatible with preparation in a radiopharmacy. Liposomes were efficiently radiolabelled (57\%) within $1 \mathrm{~h}$ with release of $\sim 12 \%$ of the radiopeptide following a 20 s exposure to US-mediated cavitation in vitro. In clonogenic studies this level of release resulted in cytotoxicity specifically in cells over-expressing the epidermal growth factor receptor (EGFR), with over $99 \%$ reduction in colony survival compared to controls. The formulation extended the circulation time and changed the biodistribution compared to the non-liposomal radiopeptide in vivo, although interestingly the biodistribution did not resemble that of liposome constructs currently 
used in the clinic. Cavitation of MBs co-injected with liposomes into tumours expressing high levels of EGFR resulted in a 2-fold enhancement in tumour uptake within 20 min. However, owing to the poor vascularisation of the tumour model used the same level of uptake was achieved without US after $24 \mathrm{~h}$. By combining acousticcavitation-sensitive liposomes with radiopharmaceuticals this research represents a new concept in achieving targeted delivery of radiopharmaceuticals.

\section{$\underline{\text { Introduction }}$}

Targeted radionuclide therapy (TRT) combines the specificity of tumour seeking carriers with the toxicity of radioisotopes[1]. There are several examples of this approach in clinical use such as ${ }^{177}$ Lutetium-Dotatate for neuroendocrine tumours[2], and ${ }^{177}$ Lutetium-prostate-specific membrane antigen (PSMA inhibitor) for metastatic castration-resistant prostate cancer[3]. However, both these strategies rely on ${ }^{177}$ Lutetium which emits high energy electrons (beta-particles) with a track length in the centimeter range which can lead to off target effects[4]. One promising strategy for TRT is the use of radionuclides that decay through electron capture and the release of short track-length $(<30 \mu \mathrm{m})$, moderately high linear energy transfer (LET) electrons that cause very localised damage and few unwanted off-target effects[4]. To cause cancer cell death these radionuclides must enter the nucleus in sufficient quantity to give rise to irreparable DNA damage. The conjugation of such radionuclides to carriers with cell-penetrating and/or nuclear localizing sequences offers a route to enhanced selectivity $[4,5]$. A previously tested example of this strategy is the use of $\left[{ }^{111} \ln \right] \ln ^{3+}$, which decays through electron capture, tagged to the HEGF peptide $\left(\left[{ }^{111} I n\right] I n-H E G F\right)$ 
which binds to the EGFR that is overexpressed by a range of solid cancers. Crucially, following binding of HEGF, the EGFR translocates to the nucleus, delivering $\left[{ }^{111} \ln \right] \ln { }^{3+}$ to the site where its decay can impact cell survival most effectively[6]. However, a notable limitation of this approach is that the small size of peptide-based radiopharmaceuticals renders them susceptible to rapid renal clearance giving rise to unfavourable circulation kinetics[7]. This can often result in insufficient accumulation of radionuclides within tumours[8]. Also in the case of small peptides reabsorption and retention in the proximal tubules after glomerular filtration can cause a high radiation dose in the kidneys therefore lowering the administered dose for small peptide radiopharmaceuticals is desirable[9]. Molecularly targeted agents such as peptides are also limited by low payloads and insufficient delivery of radioactivity to the tumour[10] as well as causing side effects such as flushing, chills, nausea, and vomiting[11]. EGFR is apparent in some of the most frequent cancers but the benefits of small agents for anti EGFR therapy plus cytotoxic agents have yet to appear[12].

118 Nanoparticles can be designed for drug delivery and may be fabricated from natural or synthetic compounds[7]. Nanoparticle delivery systems have been shown to reduce the cytotoxicity of low molecular weight chemotherapeutics and provide greater anticancer efficacy[13]. This means dosing need not be limited by the risk of severe adverse effects in non-target tissue[14]. Liposomes are a class of nanoparticle which are particularly suited to cancer therapy as they are easy to produce and can encapsulate a large payload of active therapeutics within the aqueous core of their spherical lipid bilayer. Furthermore, it is possible to surface modify liposomes with polyethylene glycol (PEG)[15] to provide protection from reticuloendothelial system

127 (RES) mediated clearance. Drug encapsulating liposomal formulations can therefore 
dramatically extend the blood circulation time compared to free drug. In turn, this may allow passive accumulation and retention in tumours through the enhanced permeability and retention (EPR) effect[16], and although the true extent of the EPR effect is contentious[17] there is pre-clinical and clinical data in support of the improved efficacy it provides. Specifically, Doxil ${ }^{\circledR}$, which was the first liposome formulation to be approved for clinical use[18, 19] demonstrates a clearance rate in humans of approximately $0.1 \mathrm{~L} / \mathrm{h}$, compared to $45 \mathrm{~L} / \mathrm{h}$ for unencapsulated doxorubicin[18, 20]. Liposomes can be produced by a variety of techniques [21, 22]. Although liposomal formulations are currently available in the clinic for delivery of chemotherapeutics[18], they have not been used clinically for the delivery of radioisotopes.

The application of liposomes to the delivery of radioisotopes therefore appears ripe for exploitation, although, there are key factors limiting the success of such a strategy. Firstly, the timescale of the conventional process of liposome formulation, loading and purification is not always compatible with the timescale and working practice required when using radioisotopes clinically. Most research has focused on decorating the surface of nanoparticles with radiopharmaceuticals [23, 24]. Although, methods to load radioactive compounds and radiopharmaceuticals into liposomes have also been developed previously, these techniques have substantial limitations that must be overcome before achieving clinical translation [23, 25-29]. For example, freeze thaw was used to load ${ }^{99 m}$ Technetium-ceftizoxime into liposomes. However, the half-life of technetium is $6 \mathrm{~h}$ and freeze thaw cycles typically take multiple hours and require

150 techniques and equipment which are not always readily available in a hospital environment[30]. Pre-loading of liposomes with a radioisotope at a manufacturing site would create transport and storage times that would compromise the amount of radioactivity available by the time of use. Methods of encapsulating radioisotopes in a 
154 more clinically viable fashion have been developed via loading of ${ }^{64}$ Copper,

$155{ }^{52}$ Manganese and $\left[{ }^{111} \mathrm{In}\right] \mathrm{In}^{3+}$ into liposomes[31-33]. Furthermore, most research

156 reported to date, has sought to encapsulate the radioisotope without providing

157 mechanisms for cancer cell targeting, release from the liposome or for trafficking of

158 the radionuclide to the nucleus once release from the liposome has occurred[34].

159 Therefore, these constructs become entirely dependent on the EPR effect for tumour 160 selectivity, with no means of either releasing the payload from the liposome or

161 ensuring penetration from the perivascular regions into and throughout the tumour 162 mass.

163

164 To address the above challenges, we describe a long-term-storage compatible 165 'platform' liposome loaded with DTPA-HEGF which can be easily post-loaded with $\left[{ }^{111} \mathrm{In}\right] \mathrm{In}{ }^{3+}$ just prior to clinical administration. Furthermore, by ensuring the liposome

167 formulation is responsive to US-mediated cavitation events, it is possible to achieve 168 temporal and spatial control of radiopharmaceutical cargo release[14]. Indeed, 169 ultrasound, a longitudinal pressure wave of frequency $>20$ kilohertz $(\mathrm{kHz})$, can be 170 efficiently delivered through the body and localised to millimetre scale tissue domains 171 when using low Megahertz $(\mathrm{MHz})$ frequencies, exposing them to alternating 172 compression and rarefaction cycles of microsecond duration. These waves can create 173 cavitation events, i.e. the expansion and collapse of a gas bubble from pre-formed 174 cavitation nuclei such as a shelled microbubble, which can be co-delivered with the 175 therapeutic into the blood stream[35]. The mechanical action of such cavitation events can increase the permeability of cell membranes, and/or selectively disrupt the

177 membranes of liposomes[14, 36, 37]. Hence, whilst in conventional drug delivery 178 systems the drug is limited to the perivascular region and transport is controlled by 
179 diffusion[36], US-mediated-cavitation used in combination with cavitation responsive 180 carriers can provide a powerful and safe mechanism to overcome tumour delivery 181 barriers by providing site-specific triggered drug release and enhanced delivery.

182

183 In this study, the aim was to determine if a cavitation sensitive liposome loaded with a 184 radiopeptide has clinical potential. We have developed a clinically relevant method of 185 afterloading $\left[{ }^{111} \mathrm{ln}\right] \operatorname{lnCl} 3$ into cavitation sensitive liposomes for conjugation to 186 encapsulated DTPA-HEGF, a tumour-targeting peptide, for enhanced 187 delivery/triggered release as represented in Figure 1. The methods used at each stage 188 are based on existing techniques, for ease of translation: $\left[{ }^{111} \ln \right] \mid \mathrm{In}^{3+}$ labelled HEGF 189 ([111 In]In-HEGF) has been tested in humans[11], the liposome formulation is based on 190 those used clinically, and MBs are also currently used in the clinics, although only as 191 US contrast agents to date. 


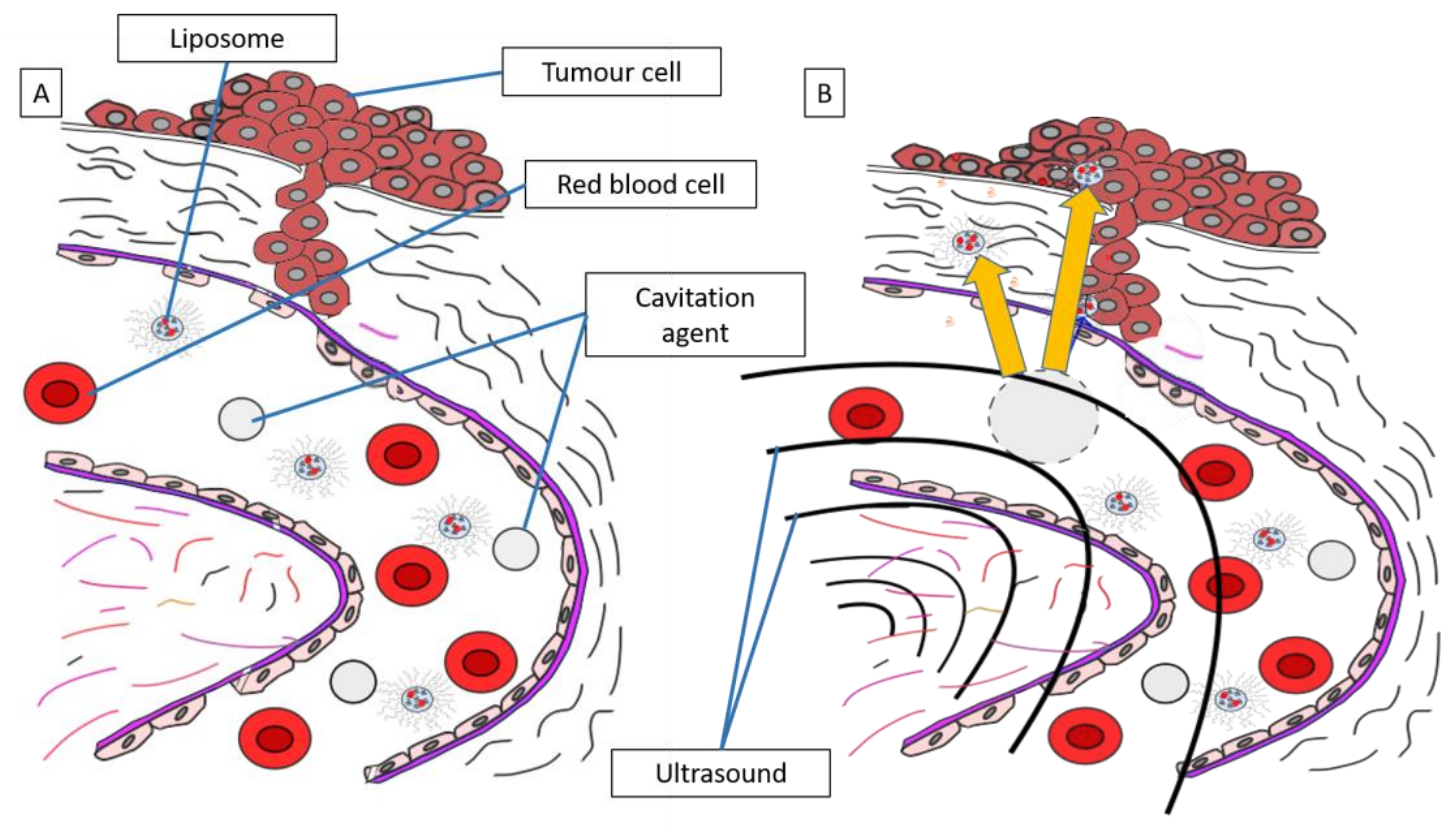

Figure 1, A) in vivo schematic of tumour cells adjacent to a blood vessel with cavitation agents and $\left[{ }^{111}\right.$ In] In-HEGF loaded liposomes and B) application of US induced cavitation to drive liposomes into tumour tissue and to release $\left[{ }^{111} I n\right] I n-H E G F$ to interact with EGFR. Objects shown in the diagram are not drawn to scale, liposomes are $175 \mathrm{~nm}$ whereas MBs are 2-8 $\mu \mathrm{m}$.

\section{Materials and Methods}

Preparation of HEGF

The conjugation of HEGF (Life Sciences, Thermo Scientific, Loughborough, UK) with diethylenetriaminepentaacetic dianhydride 98\% (DTPA) (Sigma Aldrich, Gillingham, UK) was carried out as previously described[38]. Briefly, HEGF (1 mg) was dissolved in sodium bicarbonate $(\mathrm{pH} 8.3)(400 \mu \mathrm{L})$, DTPA ( $5 \times$ molar excess) was added in DMSO (dimethyl sulfoxide) $(30 \mu \mathrm{L})$ and incubated for 45 minutes at room temperature (RT). This solution was then eluted through a Sephadex G25 (Sigma Aldrich, Gillingham, UK) column and the amount of protein recovered in each aliquot (100 $\mu \mathrm{L})$ was analysed using a Nanodrop spectrophotometer (Thermo Scientific, Wilmington, DE). HEGF was centrifuged $(12,300 \times g)$ in a molecular weight cut off $(3 \mathrm{kDa})$ 
211 ultrafiltration tube (Amicon®, Sigma Aldrich, Gillingham, UK) three times in $0.1 \mathrm{M}$

212 citrate buffer $(\mathrm{pH} 5.0)$. This was then stored at $4^{\circ} \mathrm{C}$ for later encapsulation in liposomes.

214 Synthesis of liposomes

215 All lipids were purchased from Avanti Polar Lipids, Alabaster, Alabama, USA. For the

216 liposome formulation, 1,2-dipalmitoyl-sn-glycero-3-phosphocholine (DPPC), 1-

217 tetradecanoyl-2-octadecanoyl-sn-glycero-3-phosphocholine (MSPC), 1,2-distearoylsn-glycero-3-phosphoethanolamine-N-[methoxy(polyethylene glycol)-2000] DSPEPEG(2000) at a molar ratio of (85:5:10) was used resulting in approximately $40 \mathrm{mg}$ of lipid. The chloroform was removed under high vacuum for $3 \mathrm{~h}$, using a vacuum pump (Edwards RV12, Edwards, West Sussex, UK) attached to a schlenk line (Glass Solutions, Watford, UK) via a cold trap (Glass Solutions, Watford, UK) immersed in liquid nitrogen, to leave a lipid film. Cyclohexane (2 mL) (Sigma Aldrich, Gillingham, UK) was added to the lipid film followed by vortexing, heating $\left(50^{\circ} \mathrm{C}\right)$ for 1 minute, immersion in liquid nitrogen for 2 min and freeze drying over 2 days $\left(-50^{\circ} \mathrm{C}\right.$ and 100 mTorr) (Virtis Lyostar Advantage Plus EL-85) to produce a lipid powder unattached to the glass. HEGF (see above) $(0.5 \mathrm{mg}$ ) was reconstituted in citrate buffer ( $\mathrm{pH} 5.0)$ (200 $\mu \mathrm{L}$ ) at a concentration of $10 \mathrm{mg} / \mathrm{mL}$ and this was added to the lipid powder. The lipid, HEGF solution was pipetted up and down using a $100 \mu \mathrm{L}$ pipette to break up and disperse the lipids into vesicles. Citrate buffer $(0.1 \mathrm{M}, 100 \mu \mathrm{L})$ was added and the mixture was extruded 11 times through a $100 \mathrm{~nm}$ polycarbonate membrane (Avanti Polar Lipids, Colorado, USA) at $37^{\circ} \mathrm{C}$ using a teflon mini-extruder (Avanti Polar Lipids, Colorado, USA) with 2 syringes ( $1 \mathrm{~mL})$. The extruded liposomes were then centrifuged at $12,300 \times g$ for 15 minutes in phosphate buffered saline (PBS) $(1 \times$ concentration, 
molecular weight cut-off of $30 \mathrm{kDa}$ for a minimum of three cycles to remove any excess

237 non-encapsulated HEGF. Loading efficiency was assessed via High Performance

238 Liquid Chromatography (HPLC) after SE centrifugation and ultracentrifugation (70,000

$239 \times \mathrm{g}, 30 \mathrm{~min})$ to ensure removal of excess HEGF for accurate analysis of liposome

240 loading [39]. Liposomes were then stored at $4-8^{\circ} \mathrm{C}$ for later loading with $\left[{ }^{111} \mathrm{In}\right] \operatorname{lnCl} 3$.

241 The liposome size was measured via dynamic light scattering (DLS) (Zetasizer Nano

242 ZS, Malvern Instruments, Worcestershire, UK). Empty Liposomes underwent the 243 same process but without addition of HEGF.

${ }^{111}$ In-Labelling of HEGF loaded liposomes

HEGF-Lip in PBS (pH 7.5) were incubated with $\left[{ }^{111} \ln \right] \operatorname{lnCl}_{3}(15 \mathrm{MBq})$ for $1 \mathrm{~h}$ at $38^{\circ} \mathrm{C}$,

247 following which the $\left[{ }^{111} \ln \right]$ In-HEGF-Lip were separated from free $\left[{ }^{111} \ln \right] \mid \mathrm{I}^{3+}$ by two repeats of size exclusion centrifugation (MW cut off $30 \mathrm{kDa}, 12,300 \times \mathrm{g}$ for $20 \mathrm{~min}$ ) (SE centrifugation). A CRC®-25R dose calibrator (Capintec, Inc, Florham Park, NJ) was used to measure the amount of radioactivity present in the filtrates and the collected liposomes. From mixing the HEGF liposome with $\left[{ }^{111} \ln \right] \operatorname{lnCl}{ }_{3}$ to obtaining the purified radiolabelled formulation took $2 \mathrm{~h}$.

Characterization of radiolabelled liposomes

257 To determine ${ }^{111}$ In-labelling efficiency three samples were compared: 1) Empty 258 Liposomes containing no HEGF, 2) non-purified liposomes: i.e. HEGF encapsulated within liposomes and HEGF in the solution outside the liposomes and 3) purified 260 liposomes which had HEGF encapsulated within liposomes only. These three 
samples were loaded with $\left[{ }^{111} \ln \right] \operatorname{lnCl} \mathrm{n}_{3}$ and cleaned twice using SE Centrifugation (MW cut off $30 \mathrm{kDa}, 12,300 \times \mathrm{g}$ for $20 \mathrm{~min}$ ). The amount of associated radioactivity in the filtrates and liposome solutions was then compared using the $C R C \AA-25 R$ dose 264 calibrator.

266 The radiochemical yield was determined by silica gel instant thin-layer 267 chromatography (SG-ITLC) in $0.1 \mathrm{M}$ sodium citrate buffer ( $\mathrm{pH}$ 5.2). Radioactivity 268 measurements of the silica strips were obtained using radio-TLC scanner (Eckert \& Ziegler Radiopharma, Inc., Hopkinton, MA, USA).

Transmission electron microscopy

272 Liposome morphology was observed by placing an aliquot of the liposome solution (10 $\mu \mathrm{L}$ ) on a 200-mesh Formvar-coated copper grid (Agar Scientific, Stansted, Essex), allowing it to air-dry and then negatively staining the sample with $2 \% \mathrm{w} / \mathrm{v}$ uranyl acetate and allowing further air drying. A transmission electron microscope (Tecnai T12, FEI, Hillsboro, OR) was used for image acquisition.

\section{Dynamic light scattering (DLS)}

The hydrodynamic diameter of liposomes was measured using DLS (ZetaSizer Nano, Malvern Instruments Ltd, Malvern, Worcestershire, UK). Liposome samples (10 $\mu \mathrm{L})$ were added to $990 \mu \mathrm{L}$ of PBS and each sample measured three times.

Reverse phase chromatography of liposome extracted HEGF 
286 C18 reverse phase (Supelco) column on a Shimadzu SPD 10A HPLC system

287 (Shimadzu). All samples were suspended in 7\% triton X-100 (Sigma Aldrich, 288 Gillingham, UK) and incubated for $20 \mathrm{~min}$, before buffer $\mathrm{A}$ (water with $0.1 \%$ formic 289 acid) was added to give a final volume of $700 \mu \mathrm{L}$. Samples were centrifuged at 12,300 $290 \times \mathrm{x}$ for 20 min, the supernatant was then loaded onto a C18 column in buffer A and 291 eluted from the column using eluent B ( $80 \%$ acetonitrile with $0.1 \%$ formic acid) on a 292 gradient of $0-50 \%$ B in A over 20 min. DTPA-HEGF was monitored at an absorbance of $214 \mathrm{~nm}$. Loading efficiency was determined using the following equation:

1) Loading Efficiency $(\%)=\left(\frac{H E G F \text { in Liposome }(\mu g)}{H E G F \text { stock }(\mu g)}\right) \times 100$

\section{Clonogenic assays}

300

In vitro experiments were conducted on EGFR-high MDA-MB-468 $\left(1.3 \times 10^{6}\right.$ EGFR/cell) and EGFR-low MCF7 (1 x 104 EGFR/cell) cells cultured in Dulbecco's Modified Eagle's Medium (DMEM) supplemented with 10\% fetal bovine serum (FBS) and $1 \%$ penicillin-streptomycin-glutamine (PSG). Cells were seeded in $35 \mathrm{~mm}$, high $\mu$-Dishes with a glass bottom (ibidi $\mathrm{GmbH}$, Am Klopferspitz, Planegg/Martinsried) at a concentration of $2.5 \times 10^{5}$ cells/well in $2 \mathrm{~mL}$ of supplemented DMEM. These cells were left overnight to attach. The $\mu$-Dishes were examined via light-field microscopy to determine cell attachment, the media was removed, $8 \mathrm{~mL}$ of supplemented DMEM was added and cells were exposed to combinations of $\left[{ }^{111} \ln \right] \mathrm{In}$-HEGF loaded

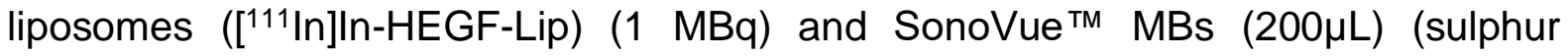


311 microbubble concentration of $3.75 \times 10^{6}$ bubbles $/ \mathrm{mL}$, prior to US exposure as outlined

312 in Table 1 below. A test group exposed to US in the absence of a MB agent (i.e. no

313 cavitation) and the presence of $\left[{ }^{111} \ln \right]$ In-HEGF-Lip was not included here because

314 previous studies with US alone with this formulation had shown it to provide no release

315 unless cavitation was instigated[14]. See Supplementary Figures 1 and 2 and

316 Supplementary Information (ultrasound system characterization) for US details and

317 characterisation. Cells were incubated for $24 \mathrm{~h}$ in the same medium, washed twice

318 with PBS, detached with Trypsin-EDTA (0.05\%), phenol red (1 mL) (Thermo Scientific,

319 Loughborough, UK) and seeded in a 6-well plate at multiple seeding densities (500,

3201000 and 2000 cells/ well). After 14 days, colonies were fixed and stained using $1 \%$

321 methylene blue mixture in methanol. Colonies were counted using a GelCount automated colony counter (Oxford Optronix, Oxford, UK).

Table 1, Samples used in clonogenic assays

\begin{tabular}{|l|c|c|c|}
\hline Sample Name & $\begin{array}{c}\text { Cavitation } \\
\text { agent }\end{array}$ & Ultrasound & $\begin{array}{c}\text { Liposomes containing } \\
{\left[{ }^{111} \text { In] In-HEGF }\right.}\end{array}$ \\
\hline Untreated & & $X$ & \\
\hline US only & $X$ & & \\
\hline MBs only & $X$ & $X$ & $X$ \\
\hline MBs + US & $X$ & & $X$ \\
\hline MBs +([111/n]In-HEGF-Lip) & $X$ & $X$ & \\
\hline MBs +([111/n]In-HEGF-Lip)+ US & & & \\
\hline
\end{tabular}

328 Liposomal release in response to ultrasound

329 Liposomes were exposed to US as described in the Supplementary Information.

$330 \quad\left[{ }^{111} \mathrm{In}\right]$ In-HEGF-Lip (5 MBq) were added to sealed plates with SV (1 mL) and exposed 
331 to US or a sham exposure at the same conditions as the clonogenic assays. After $2 \mathrm{~h}$,

$3321 \mathrm{~mL}$ of the sample was removed and centrifuged in a molecular weight cut off (30

$333 \mathrm{kDa}) \mathrm{SE}$ centrifuge tube $(12,300 \times \mathrm{g}, 20 \mathrm{~min})$ and the total percentage of radioactivity 334 in the filtrate was measured via a $\mathrm{CRC} B$-25R dose calibrator (Capintec, Inc, Florham

335 Park, NJ). The quantity of radioactivity that passed through the filters owing to 336 liposome destruction in the SE centrifugation process was also measured and 337 removed from the results.

Cellular internalisation of radioactivity

340 MDA-MB-468 or MCF7 cells were prepared in Ibidi dishes in a similar manner to the 341 clonogenic assays. Cells were exposed to [ $\left.{ }^{111} \mathrm{In}\right] \mathrm{In}-\mathrm{HEGF}-\mathrm{Lip}$ alone (1 MBq), SV alone $342(200 \mu \mathrm{L})$ or $\left[{ }^{111} \mathrm{In}\right]$ In-HEGF-Lip $(1 \mathrm{MBq})$ and SV $(200 \mu \mathrm{L})$ with US. After $2 \mathrm{~h}$ the cells were washed and trypsinised and the amount of radioactivity measured using a gamma counter (HIDEX, Lemminkäisenkatu 62 FIN-20520 Turku, Finland). To consider cell concentration the protein content of the cells was then measured using 346 the Bincinchoninic acid assay as per manufacturer's instructions (Pierce ${ }^{\mathrm{TM}}$ BCA 347 Protein Assay Kit, Thermo Fisher Scientific, MA, USA).

\section{Ethical statement}

350 All procedures were conducted in accordance with the Animals Scientific Procedures 351 Act of 1986 (UK) (Project License Numbers 30/3115 and P13B66CD9 issued by the 352 Home Office). The protocols were approved by the Committee on the Ethics of Animal 353 Experiments of the University of Oxford. All imaging was performed under 2-4\% 354 isoflurane anaesthesia delivered in oxygen enhanced room air (4\% for induction of 355 anaesthesia, 2\% for anaesthesia maintenance). Throughout imaging experiments, 
356 mice were maintained at $35-37^{\circ} \mathrm{C}$, respiration rate was monitored and all efforts were

357 made to minimize suffering. Mice were housed (5 to 6 animals per cage) in individual

358 ventilated polycarbonate solid-bottomed cages. Airflow within the cages was on a 359 positive pressure and was controlled electronically by an IVC air handling system

360 (Techniplast UK). A $12 \mathrm{~h}$ dark and light cycle was implemented with the ambient air 361 temperature set at $21^{\circ} \mathrm{C}+/-2$ with $55 \%+/-10$ humidity. All animals were provided with certified rodent diet, filtered water ad libitum, autoclaved bedding, nesting material and cage enrichment.

\section{Animal preparation}

366 Two mouse strains were used to complete the in vivo experiments. Athymic nude 367 (NU(NCr)-Foxn1nu), and Severe Combined Immunodeficiency Disease (SCID) (CB17/lcr-Prkdc<scid>/lcrlcoCrl). Athymic nude and SCID mice were selected for their immunodeficient status and ability to support growth of human tumour cells. All mice were adult females, gender was selected based on the use of a breast cancer model. Mice entered study at approximately 40 days old. The mean start weight of athymic nude mice and SCID mice was $25 \mathrm{~g}$ and $16 \mathrm{~g}$ respectively. SCID mice were used for blood lifetime studies owing to their genetic similarity to athymic nudes and smaller size for use in the required SPECT imaging cradle.

376 All cancer cells were implanted subcutaneously into the right flank of the mice. MDA377 MB-468 were injected at $~ 5$ million cells/site with $50 \%$ matrigel (Thermo Scientific,

378 Loughborough, UK) in serum free DMEM. MCF7 cells were not used in vivo due to 379 animal welfare considerations owing to their requirement for estrogen 380 supplementation. Where an indwelling cannula was used to administer contrast 
agents and treatments, a catheter (PE10, 0.28/0.64 mm internal/external diameter; Linton Instrumentation) was inserted into the lateral tail vein. Omniscan (gadodiamide,

$0.5 \mathrm{M}, 30 \mu \mathrm{L}$; GE Healthcare) was used as the contrast agent for Dynamic Contrast

Enhanced Magnetic Resonance Imaging (DCE-MRI), and SonoVue ${ }^{\mathrm{TM}}(8 \mu \mathrm{L})$ was used for contrast imaging. All injections were performed manually except for injection of contrast agent for DCE-MRI where a syringe pump was used.

In vivo biodistribution

Athymic nude mice (6 weeks old) were anaesthetised (4\% Isoflurane in air induction followed by $2 \%$ for maintenance). Animals were entered into a study when xenograft volume reached 70-100 $\mathrm{mm}^{3}$. Mice (4 per group) received either $\left[{ }^{111} \mathrm{In}\right] \mathrm{In}$-HEGF-Lip (6 $\mathrm{MBq})$ or free $\left[{ }^{111} \mathrm{In}\right] \mathrm{In}-\mathrm{HEGF}(6 \mathrm{MBq})$ via intravenous (i.v.) injection into the lateral tail vein. In both cases the volume of the injectate was $100 \mu \mathrm{L}$, with equal mass amounts of HEGF administered which was determined using HPLC. Mice were euthanised at 24 h. Xenografts and organs were harvested, weighed and the amount of radioactivity counted using a HIDEX automatic gamma counter (HIDEX, Lemminkäisenkatu 62 FIN-20520 Turku, Finland).

\section{Blood circulation time}

400 SCID mice (3 per group) were anaesthetised and received either $\left[{ }^{111} \mathrm{In}\right] \mathrm{In}$-HEGF-Lip 401 (6 MBq) or free [ $\left.{ }^{111} \mathrm{In}\right]$ In-HEGF (6 MBq) i.v. by cannula followed by a saline flush of 402 approximately $20 \mu \mathrm{L}$ to account for the dead volume in the length of the cannula.

403 Animals were placed in a bespoke mouse cradle fitted to a VECTOR 4 CT scanner 404 (MI-Labs, Heidelberglaan, Utrecht, The Netherlands) and their temperature was 405 maintained at $37^{\circ} \mathrm{C}$ using a thermostatic heating plate and monitoring via a rectal 
406

407

408

409

410

411 412 Switzerland).

413

414

415

416

417

418

419

420

421

422

423

424

425

426 Focused ultrasound in vivo

427 Mice (3 per group) received either [ $\left.{ }^{111} \mathrm{In}\right]$ In-HEGF-Lip (6 MBq) i.v. followed by SV (50

428

429

430

probe. Dynamic single photon emission tomography (SPECT) images were acquired (1 frame every $36 \mathrm{~s}$ for $2 \mathrm{~h}$ ) centred on the heart. A CT scan was acquired for anatomical information. See Supplementary Information for more details. Animals were euthanised at the end of the imaging procedure. Images were reconstructed via MI-Labs software and the signal due to $\left[{ }^{111} \mathrm{In}\right] \mathrm{In}^{3+}$ in the heart over time was analysed via PMOD software (PMOD Technologies LLC, Sumatrastrasse 25, CH-8006 Zurich,

\section{Tumour uptake of radioactivity}

Tumour uptake was determined at $24 \mathrm{~h}$ via organ harvesting as outlined above. For US enhanced delivery this was examined at 20 min via SPECT imaging as outlined above except acquiring 2 frames over 5 min in the area of the tumour and excluding the other areas of the body. The use of this 20 min time point allowed cavitation mediated uptake to be distinguished from EPR mediated uptake which may have been evident at $24 \mathrm{~h}$. The images were quantified in PMOD. The average amount of radioactivity within the tumour or an organ was obtained from mean pixel values within the ROI volume. Assuming a tissue density of $1 \mathrm{~g} / \mathrm{mL}$, the ROls were converted to $\mathrm{MBq}$ per gram and were then divided by the total administered activity to obtain the imaging ROI-derived percentage administered activity per gram of tissue (\%ID/g).

$\mu \mathrm{L})$ or $\left[{ }^{111} \mathrm{In}\right] \mathrm{In}$-HEGF-Lip $(6 \mathrm{MBq})$ followed by $\mathrm{SV}(50 \mu \mathrm{L})$ as well as tumour targeted

US. The total injection time for the combined treatment of liposomes and SV was 90 \pm 20 s. US ( $1 \mathrm{MHz}, 1 \mathrm{MPa}, 30 \%$ duty cycle, $10 \mathrm{~ms}$ pulse period) was applied to tumours 
431 for 5 min. US was applied after injection of SV was completed. Tumours were treated 432 when they reached an approximate volume of $70-100 \mathrm{~mm}^{3}$. A mouse was placed on an acoustically transparent mylar bed above a water bath thermostatically controlled

434 at $37^{\circ} \mathrm{C}$. A $1.0 \mathrm{MHz}$ center frequency US source was positioned in the water directly underneath the animal (Supplementary Figure 3). The focus of the source was aligned to a spot marked on the mylar bed where the target tumour would be placed. The center of the transducer housed a 7.5 MHz US transducer (Panametrics V320-SU-F 1.75PTF, Olympus NDT, Essex, UK) that was used as a passive cavitation detector (PCD). Receiver signals were high-pass filtered (F5081-2P0, Allen Avionics, Mineola, NY, USA), preamplified (SR445A, SRS, Sunnyvale, CA, USA), digitized (Handyscope HS3, TiePie Engineering, Netherlands) and streamed to a laptop computer disk. For analysis of cavitation activity, PCD time series data sets were processed in Matlab (Mathworks, Natick, MA, USA) using Welch's method for power spectrum calculation, implemented with temporal windows of $80 \mu$ s $(2.5 \%$ of typical source drive pulse length) with $50 \%$ window overlap. Before therapy was started, a small amount of centrifuged US coupling gel (Aquasonic 100, Parker Laboratories, Fairfield, NJ) was applied to the mylar at the mark where the tumour would be placed. Centrifuging the coupling gel ensured no air bubbles were present.

$\underline{\text { Results and Discussion }}$

452

Liposome characterisation

454 Liposome modal diameter, as assessed by DLS (Supplementary Figure 4A, B), was 455 found to be $175 \mathrm{~nm}$ with a polydispersity index of 0.15 . Transmission electron 
456 microscopy images were in accordance with this size range, with a spherical shaped 457 lipid shell evident, as shown in Figure 2A. Similar size and polydispersity readings 458 were obtained for all liposome formulations, regardless of the presence or absence of 459 HEGF or $\left[{ }^{111} \ln \right] \ln ^{3+}$ (supplementary Figure $4 \mathrm{C}$ ).

460

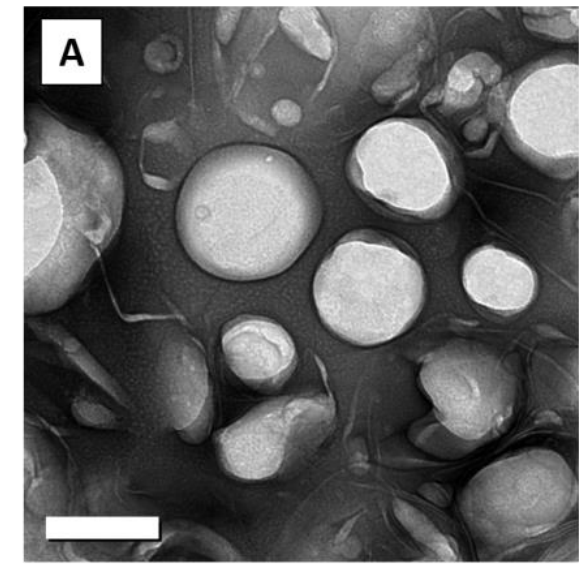

C

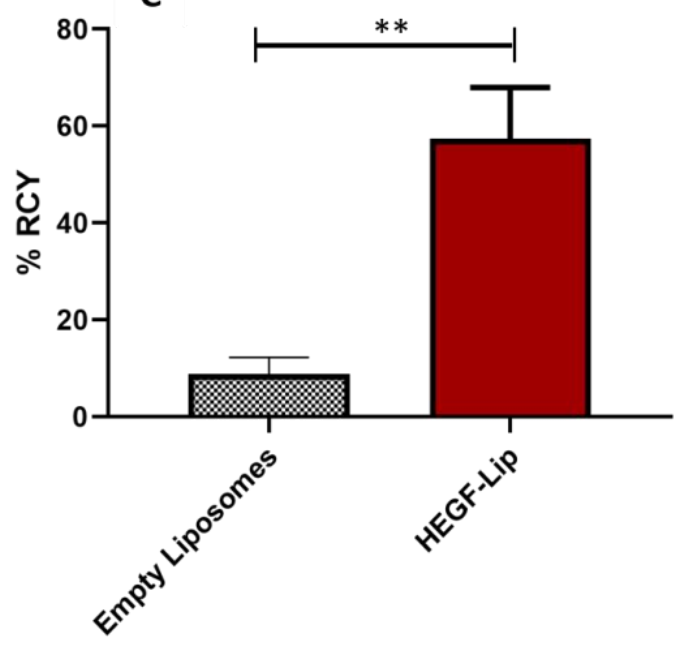

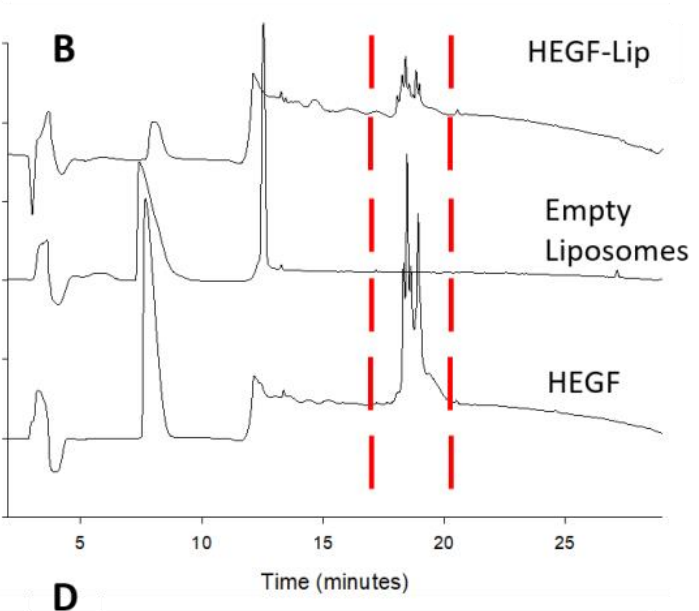

D

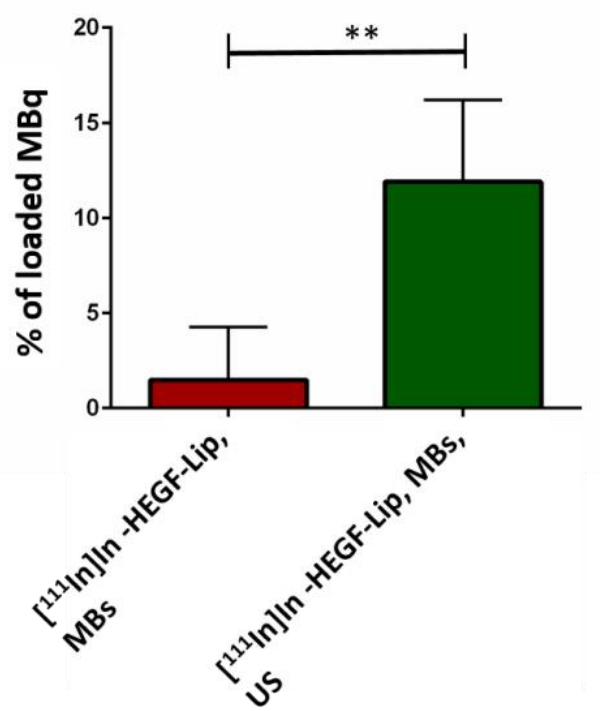

461

462 Figure 2, Characterisation of liposomes. A) Transmission electron microscopy confirming the size and showing the morphology of the HEGF-Lip (scale bar $=100 \mu \mathrm{m})$. B) High Performance Liquid 464 Chromotography analysis of HEGF, Empty Liposomes and HEGF-Lip cleaned by ultracentrifugation. C) $465{ }^{111}$ In-labelling efficiency of empty liposomes compared to liposomes containing HEGF, after addition of $466\left[{ }^{111} \mathrm{In}\right] \mathrm{InCl}_{3}(11 \mathrm{MBq})$ and 2 cycles of ultrafiltration $(n=3)$. D) Analysis of ${ }^{111}$ In-HEGF release from $467 \quad\left[{ }^{111}\right.$ In] In-HEGF-Lip, with MB, with or without US application $(n=5) . p<0.005$ by students unpaired two 
tailed t-test. Error bars represent standard deviation. $Y$ axis on D represents $\%$ of $6.3 \mathrm{MBq}$ loaded within the HEGF-lip of $C$.

\section{Passive loading of liposomes with HEGF}

The level and efficiency of HEGF loading into liposomes and the success of ultracentrifugation and SE centrifugation in removing free non-encapsulated HEGF was assessed using HPLC. 15\% of the added HEGF was loaded into the liposomes The dashed red lines in Figure 2Figure 2B shows the peak at 18 min that is present in the profile for HEGF alone and the profile for HEGF-Lip but absent for control 'Empty Liposomes' to which no HEGF was added. This level of loading is in-line with that typically achieved by similar passive loading techniques[40]. This resulted in a peptide to lipid ratio of approximately $1: 315 \mathrm{mg}$, considering lipid loss during cleaning. This is a limitation for all passive protein loading techniques which are inefficient because the liposomes cannot entrap $100 \%$ of the surrounding protein solution[41]. Cleaning techniques may also result in loss of the lipids. Importantly, the purification method employed in this study does allow the recovery and potential re-use of nonencapsulated HEGF[42]. The quantities of lipid and HEGF combined would be acceptable for human use if scaled-up based on calculations from Barenholz and Vallis et al.[11, 18].

\section{Indium-111 labelling of liposomes}

Having established that HEGF could be loaded into the liposomes the question of whether the internalised peptide could then be rapidly and efficiently radiolabelled with $\left[{ }^{111} \operatorname{In}\right] \mid \mathrm{In}^{3+}$ was investigated. Figure $2 \mathrm{C}$ shows that of the $11 \mathrm{MBq}$ of $\left[{ }^{111} \ln \right] \operatorname{lnCl} 3$ added to control empty liposomes (i.e. no HEGF), less than $1 \mathrm{MBq}$ was retained after 
washing. Similarly, when liposomes were loaded with HEGF and $\left[{ }^{111} \ln \right] \operatorname{lnCl} \mathrm{I}_{3}$ added

494

495

496

497

498

499

500

501

502

503

504

505

506

507

508

509

510

511

512

513

514

515

516

517

518

before the non-encapsulated HEGF was removed by purification ('internal and external') the amount of radioactivity (2 MBq) was low (Supplementary Figure 5). This emphasises the need to remove free HEGF through purification after the passive loading step. In contrast when $\left[{ }^{111} \ln \right] \operatorname{lnCl} 3$ was added to purified HEGF liposomes $>6$ $\operatorname{MBq}(57 \% \pm 10)$ of the $\left[{ }^{111} \ln \right] \ln ^{3+}$ was encapsulated. Furthermore, $>75 \%$ of the $\left[{ }^{111} \mathrm{In}\right] \mathrm{ln}{ }^{3+}$ remained incorporated after the liposomes were added to serum at $37^{\circ} \mathrm{C}$ for $5.5 \mathrm{~h}$ (Supplementary Figure 5D). When these purified products were analysed by SGITLC (Supplementary Figure 6) it was apparent that of the $1 \mathrm{MBq}$ associated with the empty liposomes, 34\% was incorporated. This radiolabelling is possible because $\left[{ }^{111} \ln \right] \operatorname{lnCl}{ }_{3}$ can pass passively through the lipid shell, however, in the absence of HEGF, there is no mechanism to retain $\left[{ }^{111} \mathrm{In}\right] \mathrm{InCl}_{3}$ inside the liposome and so over time it can diffuse back out. In contrast, of the $6 \mathrm{MBq}$ associated with HEGF-DTPA liposomes $93 \%$ remained at the SG-ITLC origin demonstrating efficient and robust incorporation within the liposomes.

To probe the nature of the $\left[{ }^{111} \ln \right] \mathrm{In}^{3+}$ association with empty liposomes further, Triton X 100 was added to the empty liposomes and SG-ITLC analysis repeated (Supplementary Figure 7). Notably, post-Triton addition, $15 \%$ of the $1 \mathrm{MBq}$ still remained at the origin. This suggests that a low level of $\left[{ }^{111} \ln \right] \operatorname{lnCl}{ }_{3}$ interacts with the liposomal surface but is not internalised or retained. It is possible that this observation results from a transient interaction between the phosphate groups on the phospholipids of the liposome and the $\left[{ }^{111} \ln \right] \operatorname{lnCl}_{3}[43]$. 
$\left[{ }^{111} \mathrm{In}\right] \mathrm{In}$-HEGF-Lip were tested for US enhanced release in the presence of cavitating

521 MBs. The amount $\left[{ }^{111} \mathrm{In}\right] \mathrm{In}-\mathrm{HEGF}$ that was released from liposomes that were exposed

522 to MBs and US (i.e. cavitation) for $20 \mathrm{~s}$ was $12 \pm 4 \%$ of the total. In contrast, in the

523 absence of cavitation only $1.5 \pm 2 \%$ of the cargo was released, as shown in Figure

524 2Figure 2D. Graham et al. reported $20-30 \%$ release of doxorubicin from liposomes using a similar setup. Lentacker et al. reported 'significant' release of doxorubicin from

526 liposomes attached to MBs and this resulted in a doubling of cell kill relative to

527 liposomes that were not exposed to US[14, 44]. However, these examples may not be directly relevant as they concern the release of a drug (doxorubicin) with a much lower molecular weight than $\left[{ }^{111} \mathrm{In}\right] \mathrm{In}-\mathrm{HEGF}$. The release of $12 \pm 4 \%$ of $\left[{ }^{111} \ln \right] \mathrm{In}-\mathrm{HEGF}$ would be interesting to examine clinically in terms of the added cost of ultrasound relative to improvements in patient treatment to $\left[{ }^{111} \mathrm{In}\right] \mathrm{In}$-HEGF-Lip alone. Attachment of $\left[{ }^{111} \mathrm{In}\right] \mathrm{In}$ HEGF-Lip to MBs could improve release and therapeutic efficacy[45] but would also add complexity to the manufacture and purification process perhaps precluding facile synthesis in a hospital setting. Cavitation caused release of $\left[{ }^{111} \ln \right] \mathrm{In}-\mathrm{HEGF}$ from the liposomes in vitro, but in vivo the ultrasound would also cause enhanced delivery[36] and improved endothelial opening[46] the combination of which is likely improve costto-benefit. It is important to note that whilst the in vitro experiments were limited to a

53820 second ultrasound exposure time, in vivo experiments allow for replenishment of MBs and so longer exposure times on the order of minutes can occur. With regard to safety, recent research has shown that the observed extent of cavitation would have

541 minimal impact on red blood cells [47]. 

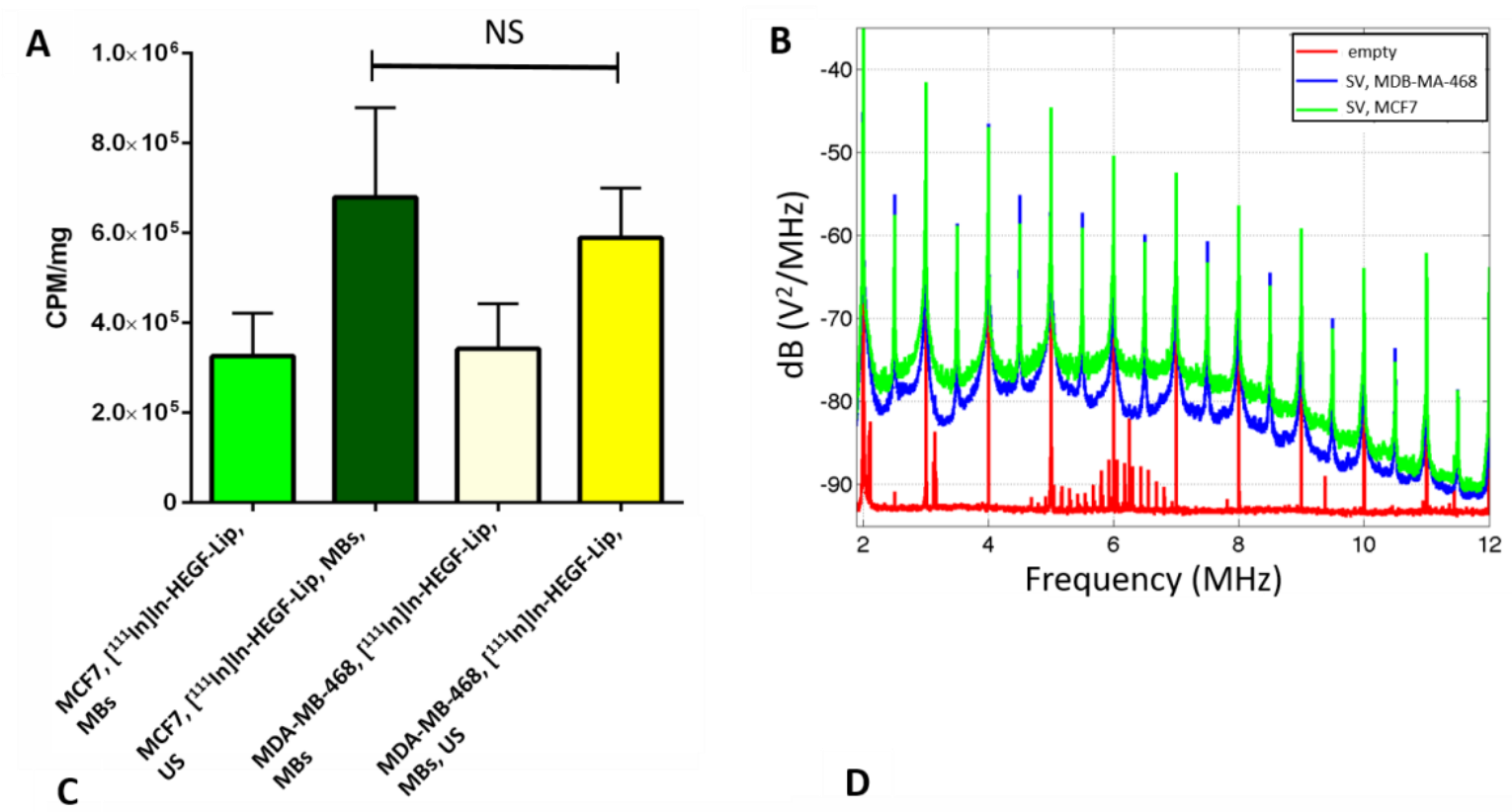

C
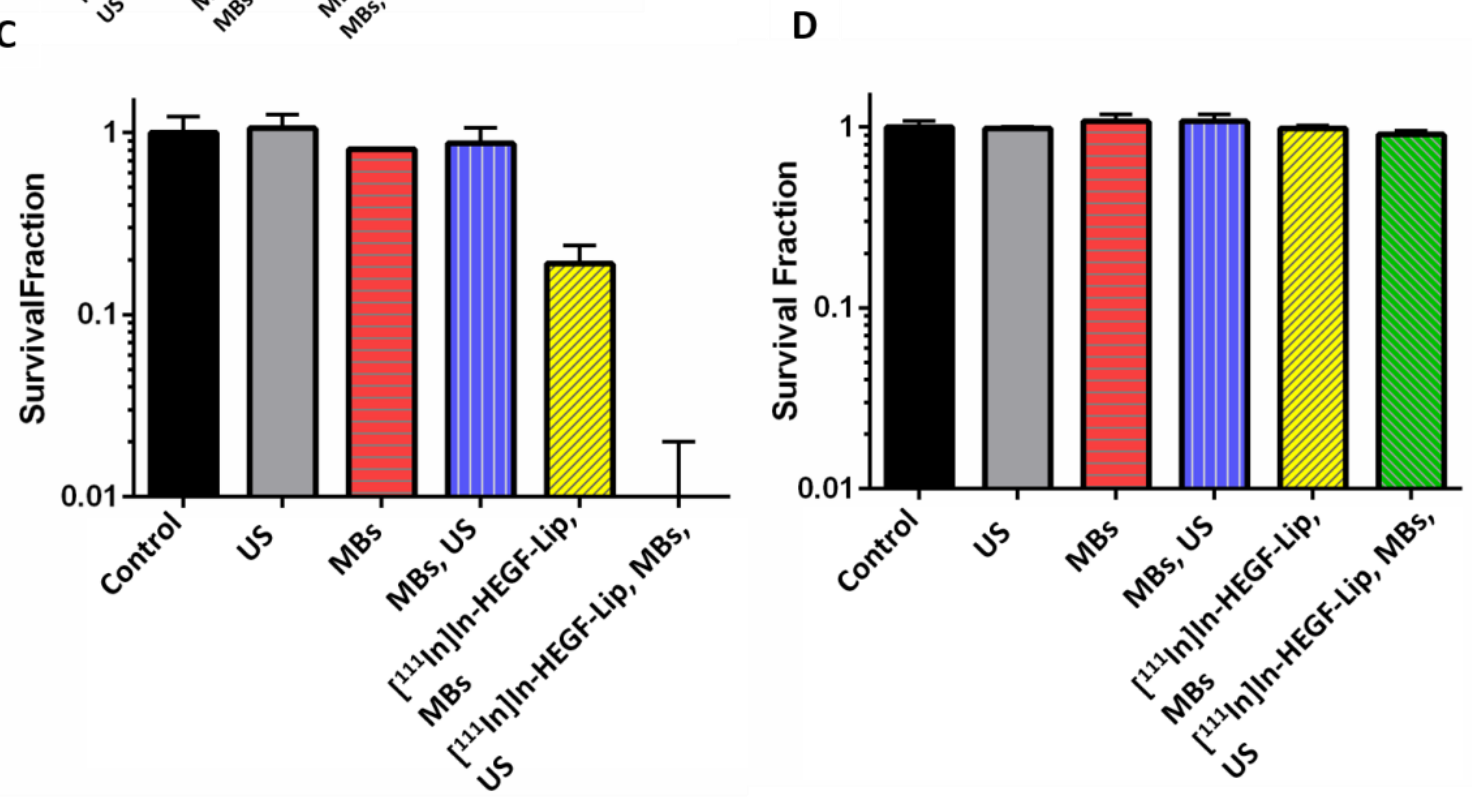

543

544 Figure 3, Assessing delivery of [ ${ }^{111}$ In] In-HEGF to cells in vitro. A) Counts per minute (CPM) per mg of protein for MCF7 cells and MDA-MB-468 cells exposed to [111/n]In-HEGF-Lip and MBs with and without US for 20 seconds, followed by incubation for $2 h$ ( $n=3 ; p>0.05$; two tailed student's $t$ test). B)

547 Examples of PCD power spectra averaged over the 20-second US exposure period for an empty dish

548 (control containing only media and no cells) and two cell lines with SV and [111In]In-HEGF-Lip. Relative

549 to the control, the latter two spectra show elevated responses at integer and half-integer multiples of

550 the drive frequency $(1.0 \mathrm{MHz})$ indicating nonlinear bubble vibration, along with broadband elevation

551 at all frequencies, suggesting bubble collapse. C) Comparison of colony survival for MDA-MB-468 cells;

552 controls, cells exposed to US only, MBs only, MBs and US, $\left[{ }^{111} \ln \right] \ln$-HEGF-Lip and MBs, $\left[{ }^{111} / n\right] I n-H E G F-$

553 Lip and MBs and US. D) Comparison of MCF7 cells following exactly the same exposure conditions ( $n=$ 554 3). 
555

556 Cellular uptake of $\left[{ }^{111} \mathrm{In}\right] \mathrm{In}$-HEGF-Lip with and without US was measured after incubation of both cell lines for $2 \mathrm{~h}$. A difference in uptake was noted to be dependent on US application, and not on receptor availability (Figure 3A). Incubation of MDAMB-468 cells with $\left[{ }^{111} \mathrm{In}\right]$ In-HEGF-Lip, MBs and exposure to US gave an increase in

$560 \mathrm{CPM} / \mathrm{mg}$ of protein relative to cells exposed to $\left[{ }^{111} \mathrm{In}\right] \mathrm{In}-\mathrm{HEGF}$-Lip and MBs but no US.

561 The same finding was evident with MCF7 cells which have low expression of EGFR.

562 No statistical difference between the uptake into the two cell lines when exposed to 563 US was observed $(P>0.05)$. To exclude the possibility that interaction between MB 564 cavitation and liposomes could be the cause of non-specific association with both cell 565 lines, their exposure to liposomes and to cavitation events were temporally separated. 566 Cells were incubated with $\left[{ }^{111} \mathrm{In}\right] \mathrm{In}$-HEGF-Lip, and a media change and wash 567 performed after $2 \mathrm{~h}$, followed by addition of MBs with or without US application. No difference in uptake was then observed with and without US (Supplementary Figure $8 \mathrm{~A}$ and $\mathrm{B})$. The results indicate that the US is not only causing release of $\left[{ }^{111} \mathrm{In}\right] \mathrm{In}$ -

570 HEGF as shown in Figure 2D but also potentially driving $\left[{ }^{111} \ln \right]$ In-HEGF-Lip and 571 released $\left[{ }^{111} \mathrm{In}\right] \mathrm{In}-\mathrm{HEGF}$ through the cell membrane into the cell cytoplasm regardless 572 of cell EGFR density[48]. This is in accordance with a 'sonoporation' effect reported 573 previously in several in vitro experiments[49-51]. To explore this further cell 574 compartments were examined after $24 \mathrm{~h}$ (Supplementary Figure 9C) and showed that 575 the signal for MDA-MB-468 cells was detected in the cytoplasm and the nucleus, 576 whereas for MCF7 there is negligible signal detected in both fractions indicating the sonoporation has a transient effect for MCF7. There is also reduced exposure on the cell membrane. This agrees with mechanism of cytotoxicity for $\left[{ }^{111} \ln \right] \ln -\mathrm{HEGF}$ as 579 reported by Reilly et al. which exploits the normal internalisation pathway for peptide 
580 growth factors after their binding to cell surface receptors[6]. This pathway involves

581 internalisation of growth factors and their receptors into cytoplasmic vesicles for

582 proteolytic degradation and potential nuclear translocation[52]. The ultrasound

583 causes liposomal release and increased uptake into cells regardless of cell receptor

584 density, but for [ $\left.{ }^{111} \mathrm{In}\right]$ In-HEGF to have a cyctotoxic impact it requires the pathway to 585 nuclear transcription. This could possibly require a re-entry mechanism of $\left[{ }^{111} \operatorname{In}\right] \ln$ HEGF and requires further exploration for the impact of ultrasound and nanoparticles on cellular uptake and cytotoxicity.

588

589 Cell survival

590 Using clonogenic assays it was shown that in the absence of $\left[{ }^{111} \ln \right]$ In-HEGF-Lip the physical stimulus of US and addition of MBs, either alone or in combination, had minimal impact on cell survival in either cell line as the survival fraction (SF) was $>85 \%$. In contrast, [ $\left.{ }^{111} \mathrm{In}\right]$ In-HEGF-Lip were highly cytotoxic to MDA-MB-468 cells when used in combination with MBs and US. Whereas SF was maintained at 1 for all conditions in MCF7 cells SF was approximately 100-fold lower in MDA-MB-468 cells exposed to MB plus $\left[{ }^{111} \mathrm{In}\right] \mathrm{In}$-HEGF-Lip with US. (Figure 3C and D). Cavitation events triggered by MBs in the presence of US (Figure 3B) may cause release of [ $\left.{ }^{111} \ln \right] \ln$-HEGF from liposomes allowing receptor mediated uptake to take place. The in vitro data shown here and previous studies by Graham et a\[14] indicate that uptake of released payload 600 is likely to be occurring. However, the work of Lentacker et al[49] demonstrates that passage of the whole liposomes into cells is also a possibility. However, MBs with $\left[{ }^{111} \mathrm{In}\right] \mathrm{In}$-HEGF-lip and US only reduced the viability of EGFR overexpressing cells without impacting cells with minimal EGFR expression (Figure $3 \mathrm{C}$ and D). 
605 Exposure of MDA-MB-468 cells to [111In]In-HEGF-Lip and MBs resulted in $80 \pm 5 \%$ 606 cell death even in the absence of US. One possible explanation for this observation is 607 that liposome degradation of the liposomes occurred during the $24 \mathrm{~h}$ incubation at $60837^{\circ} \mathrm{C}$, with release of $\left[{ }^{111} \mathrm{I} \mathrm{n}\right] \mathrm{In}-\mathrm{HEGF}$ which only impacted MDA-MB-468 cells owing to 609 higher EGFR expression.

610

611 Cavitation only occurred when MBs were present in solution at the time that US was

612 applied (Figure 3B). No difference in toxicity was observed between MDA-MB-468 613 cells and MCF7 cells when MBs were cavitated in the presence of US but absence of $614\left[{ }^{111} \mathrm{In}\right]$ In-HEGF-Lip (Figure $3 \mathrm{C}$ and D). The cavitation signal was highest during the first $6151.5 \mathrm{~s}$ after which the cavitation signal markedly reduced by a factor of $4-6$ and by an 616 order of magnitude after $20 \mathrm{~s}$ (Supplementary Figure 9). It is possible that $20 \mathrm{~s}$ of US 617 exposure is longer than required in this in vitro set-up. 
620

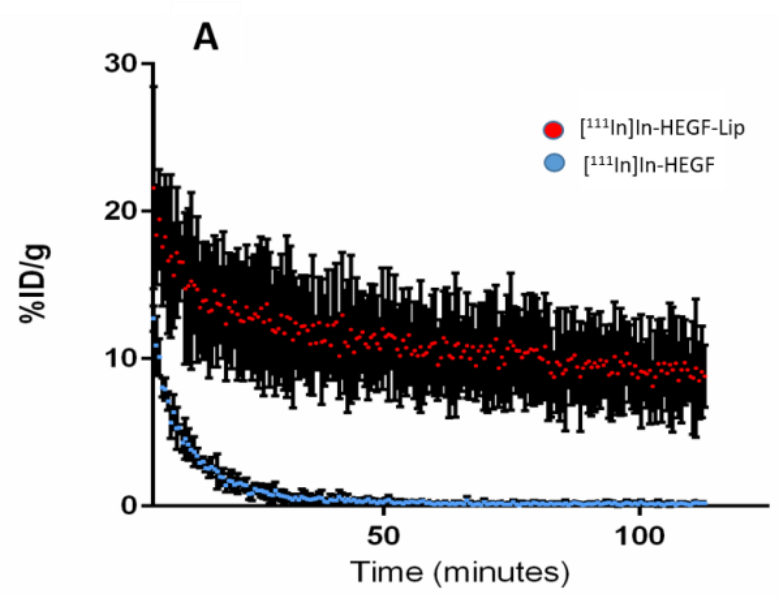

B
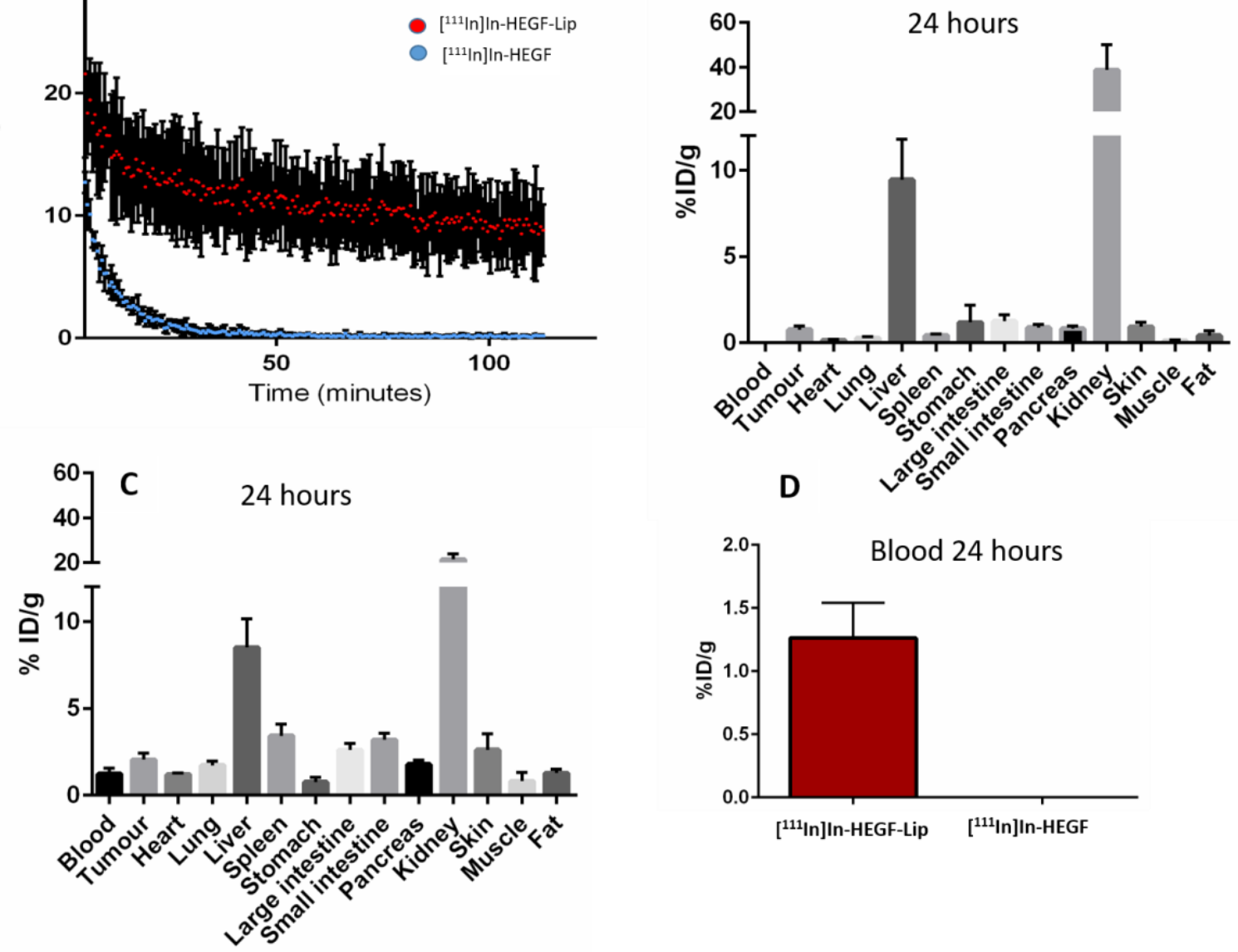

621

Tumour 24 hours

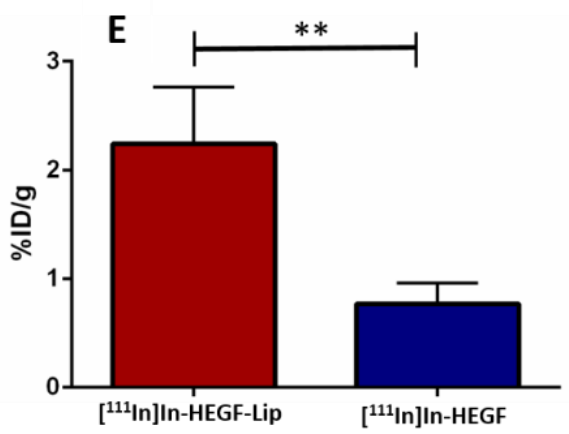

622 Figure 4, Distribution and pharmacokinetics in a murine xenograft model in percentage of injected

623 dose per gram (\%ID/g), A) blood lifetime of [111/n]In-HEGF-Lip (red) and free [111/n]In-HEGF (blue) over

$6242 \mathrm{~h}$ from SPECT imaging $(n=3)$, B) Biodistribution of free $\left[{ }^{111} \ln \right] \operatorname{In}$-HEGF $(n=4)$ and C) $\left[{ }^{111} \ln \right] \ln$-HEGF-Lip $625(n=4) 24 \mathrm{~h}$ post injection, D) levels of radioactivity in the blood at $24 \mathrm{~h}$ from scintillation counting. ( $n=4)$ 626 standard deviation shown, and E) Tumour uptake at $24 \mathrm{~h}$ with unpaired student $t$-test, $* *=p<0.005$. 
628 The $\left[{ }^{111} \mathrm{In}\right]$ In-HEGF-Lip were tested in vivo to examine biodistribution and 629 pharmacokinetics. [ $\left.{ }^{111} \mathrm{In}\right]$ In-HEGF-Lip was shown to have an extended blood 630 circulation time relative to free $\left[{ }^{111} \ln \right]$ In-HEGF. As shown in Figure 4A the amount of $631\left[{ }^{111} \mathrm{In}\right] \mathrm{In}-\mathrm{HEGF}$ decreased rapidly to $0.1 \pm 0.1 \% \mathrm{ID} / \mathrm{g}$ within thirty minutes, whereas 632 after 2 h, $10 \pm 5 \%$ ID/g of liposomes were still present in the blood. However, the profile 633 for the liposomes shows a rapid decrease over the course of the first ten minutes post 634 injection (p.i.). The decay for [ $\left.{ }^{111} \mathrm{In}\right] \mathrm{In}-\mathrm{HEGF}$ was exponential with a blood half-life of $635174 \mathrm{~s} \pm 19 \mathrm{~s}$. The blood half-life for the $\left[{ }^{111} \mathrm{In}\right] \mathrm{In}-\mathrm{HEGF}-\mathrm{Lip}$ was bi-exponential with a 636 rapid phase, $101 \mathrm{~s} \pm 61 \mathrm{~s}$, followed by a slower phase $\sim 50 \mathrm{~min} \pm 19 \mathrm{~min}$. This is likely 637 to represent a distribution phase followed by an elimination phase[53] but it may also 638 be the consequence of release of up to $25 \%$ of the payload due to temperature and serum mediated destabilization (supplementary figure 5), or also possibly due to a small quantity of free $\left[{ }^{111} \mathrm{In}\right]$ In-HEGF which was not removed during the cleaning 641 process. Notably, after $24 \mathrm{~h}\left[{ }^{111} \mathrm{In}\right] \mathrm{In}-\mathrm{HEGF}-\mathrm{Lip}$ is still detectable in the blood whereas $642\left[{ }^{111} \mathrm{In}\right] \mathrm{In}-\mathrm{HEGF}$ is below detectable limits (Figure 4D).

$644\left[{ }^{111} \mathrm{In}\right] \mathrm{In}-\mathrm{HEGF}-\mathrm{Lip}$ results in a change in the $24 \mathrm{~h}$ biodistribution that differs from that 645 of free $\left[{ }^{111} \mathrm{In}\right] \mathrm{In}-\mathrm{HEGF}$. As shown in Figure 4B the majority of $\left[{ }^{111} \mathrm{In}\right] \mathrm{In}-\mathrm{HEGF}$ 646 accumulated in the kidneys and liver whereas for the liposomal formulation (Figure 6474 C) renal clearance reduced by half. Interestingly there is no increased signal in the 648 liver for this liposomal construct, which was unexpected and different to other clinically 649 available liposome formulations[53]. The biodistribution was also confirmed by $24 \mathrm{~h}$ 650 SPECT images where signal for $\left[{ }^{111} \ln \right]$ In-HEGF and $\left[{ }^{111} \ln \right] \ln$-HEGF-Lip is detectable 651 in the kidneys and the liver (Supplementary Figure 10). Using scintillation counting of 
organs ex vivo, most organs have double the amount of accumulated $\left[{ }^{111} \ln \right] \ln { }^{3+}$ when the liposomal formulation was used relative to the free radiopeptide. This effect was also evident in the tumour as the liposomal formulation delivered over twice the amount $(2.2 \pm 0.5 \% \mathrm{ID} / \mathrm{g})$ relative to free $\left[{ }^{111} \mathrm{In}\right] \mathrm{In}-\mathrm{HEGF}(0.8 \pm 0.2 \% \mathrm{ID} / \mathrm{g})$ as shown in

Figure 4E. The tumour to blood ratio was 1.77 for $\left[{ }^{111} \mathrm{In}\right] \mathrm{In}-\mathrm{HEGF}-\mathrm{Lip} 24 \mathrm{~h}$ after injection, however, no ratio could be calculated for the ${ }^{111}$ In-HEGF because the quantity in the blood was negligible. The tumour to muscle ratio was $3.15 \pm 1.8$ for the $\left[{ }^{111} \mathrm{In}\right] \mathrm{In}-\mathrm{HEGF}-\mathrm{Lip}$ and $5.69 \pm 2.4$ for $\left[{ }^{111} \mathrm{In}\right] \mathrm{In}-\mathrm{HEGF} 24 \mathrm{~h}$ after injection but, these figures were not statistically different, indicating that $\left[{ }^{111} \ln \right] \ln -$ HEGF-Lip is distributed more widely throughout the organs resulting in a higher background level. For $\left[{ }^{111} \mathrm{In}\right] \mathrm{In}$-HEGF-Lip at $24 \mathrm{~h}$ approximately $1 \% \mathrm{ID} / \mathrm{g}$ remains in the blood and 663 approximately $2 \% \mathrm{ID} / \mathrm{g}$ is recovered from the tumour. There is a possibility that the blood load may over-estimate the level being assigned to tumour accumulation. However, it should be noted that the tumours are very poorly perfused (Supplementary Figure 11) and blood quantity in the tumour may be negligible. This is evidenced by the fact that at 20 min (when blood activity levels were $15 \times$ higher than at $24 \mathrm{~h}$ ) the tumour associated level without ultrasound (Figure 5A) was only $1 \%$. However, the extended circulation time and the change in biodistribution are strikingly different for $\left[{ }^{111} \mathrm{In}\right] \mathrm{In}$-HEGF-Lip relative to $\left[{ }^{111} \mathrm{In}\right] \mathrm{In}-\mathrm{HEGF}$ alone. Further investigation of the role of

671 the RES in the capture, degradation and excretion of the liposomes would be useful 672 in understanding and optimising the PK of this formulation to allow further enhancements of passive and potentially active delivery to tumours.

674

675 The $~ 20 \%$ uptake in the kidneys from $\left[{ }^{111}\right.$ In]ln-HEGF-Lip (Figure 5C) was unexpected 676 even though it represents only half the amount observed for $\left[{ }^{111} \ln \right]$ In-HEGF. One 
677 possible explanation is slow release of the $\left[{ }^{111} \mathrm{In}\right] \mathrm{In}-\mathrm{HEGF}$ from inside the liposome 678 over time. Another possibility is release of $\left[{ }^{111}\right.$ In]In-HEGF bound to the liposome 679 surface over time. However, this is less likely because the liposomes are PEGylated 680 and as such there are no potential binding sites for HEGF. Furthermore, the 681 pharmacokinetic profile and biodistribution of the liposomes did not match those 682 produced by a liposome formulation with externally bound [111 In]In-HEGF[54] and 683 there was no correlation of in vitro cellular uptake of [ ${ }^{111}$ In]ln-HEGF-Lip and EGFR 684 density (Figure 3A) which would be expected of liposomes with surface bound [111 In]In685 HEGF.

686

687

688

689

690

691

692

693

694

695

696

697

698

699

700

701 
704
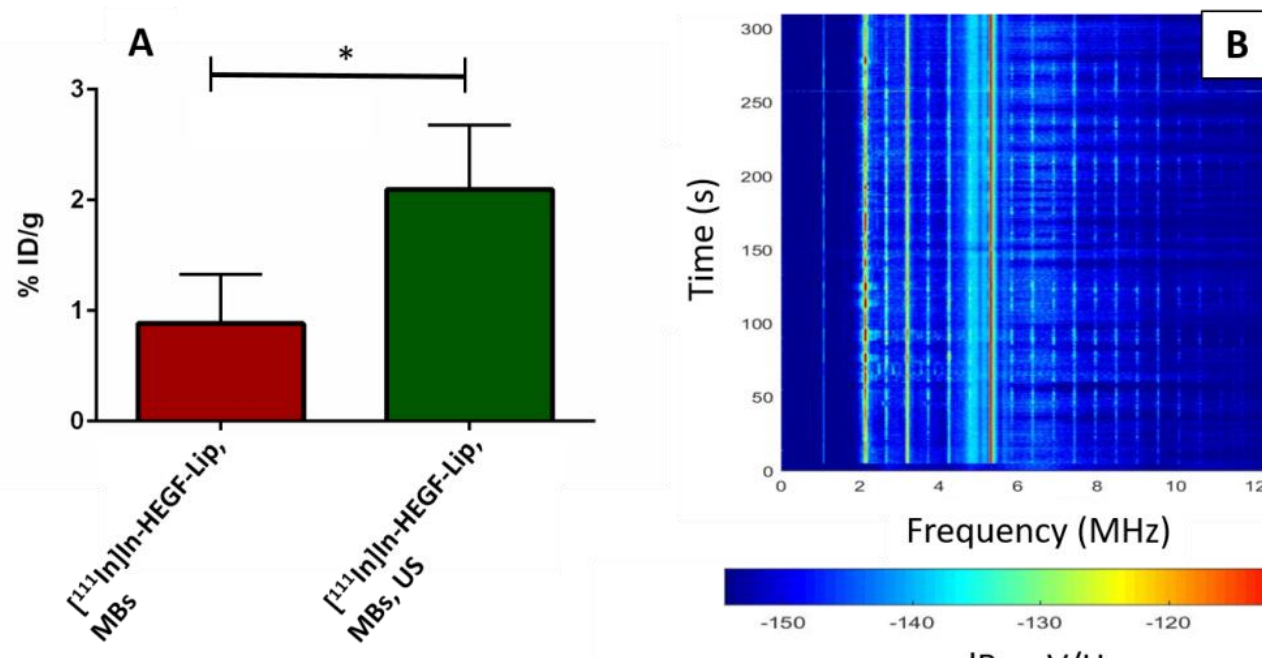

Frequency $(\mathrm{MHz})$

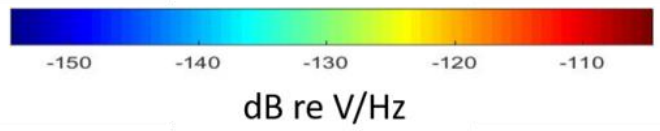

705
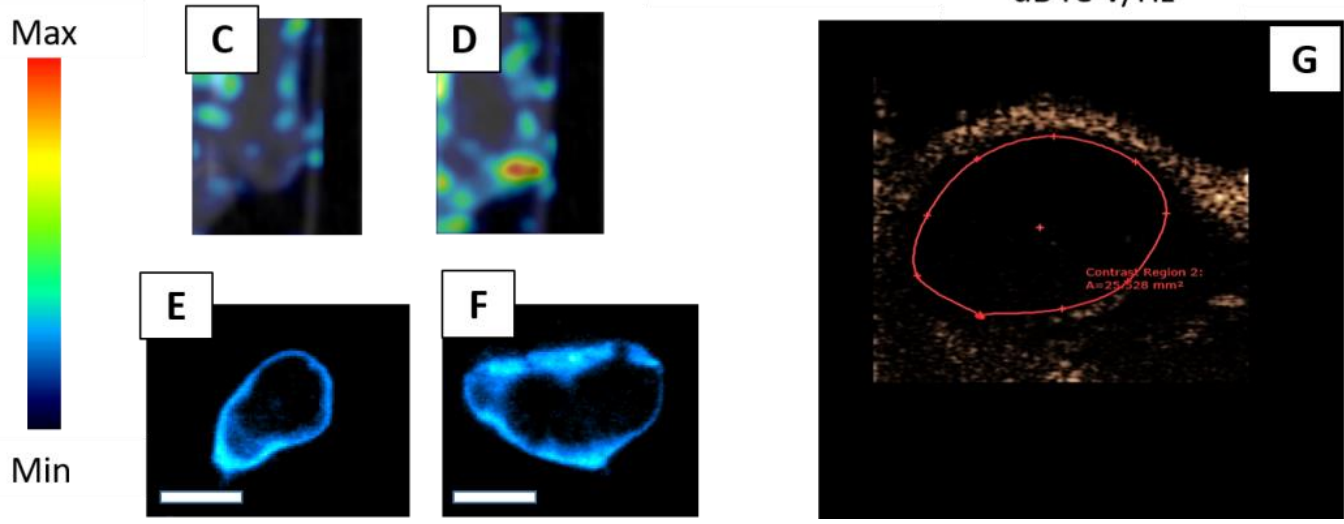

706

Figure 5, US enhanced tumour uptake of [111/n]In-HEGF-Lip. A) Tumour uptake 20 minutes after injection of $\left[{ }^{111}\right.$ In] In-HEGF-Lip, MBs comparing US to non-US treated tumours, unpaired student $t$-test $p<0.05, n=3$, error bars represent standard deviations. B) Acoustic data collected during treatment providing validation of cavitation occurrence. C) Representative SPECT images of mouse tumour area after injection of $\left[{ }^{111}\right.$ In] In-HEGF-Lip without US and D) with US. E) Autoradiography after $24 h$ examining distribution of indium within a tumour section following treatment with [111/n]In-HEGF-Lip and MBs or F) [111 In] In-HEGF-Lip and MBs and US (scale bar: $3 \mathrm{~mm}$ ), G) contrast enhanced US image of a MDA-MB-468 tumour in an athymic nude mouse with MBs.

715 Having established that the $\left[{ }^{111}\right.$ In]In-HEGF-Lip formulation provided more a favourable PK compared to free $\left[{ }^{111} \mathrm{In}\right] \mathrm{In}$-HEGF, the impact of tumour directed US exposure on 
717 the liposomes was studied. Intravenous [ $\left.{ }^{111} \mathrm{In}\right] \mathrm{In}$-HEGF-Lip and MB delivery to tumour

718 (20 min p.i.) with the application of US was $2.1 \pm 0.5 \% \mathrm{ID} / \mathrm{g}$ versus $0.9 \pm 0.4 \% \mathrm{ID} / \mathrm{g}$ for

$719\left[{ }^{111} \mathrm{In}\right] \mathrm{In}$-HEGF-Lip and MBs alone $(\mathrm{n}=3, P<0.05)$. Cavitation was confirmed via PCD

720 data (Figure 5B) specifically in the appearance of ultraharmonics (half integer

721 multiples of the FUS frequency) and broadband noise which are well-known

722 characteristics of nonlinear bubble behaviour in an US field[55]. In all US experiments with MBs, the total (ultraharmonic + broadband) cavitation level was at least five times

724 greater than the root mean square background noise. Such acoustic feedback 725 provides confidence that cavitation events occurred within the tumour at the time of 726 liposome transition through the tumour vasculature. SPECT images of mice tumours 727 taken at 20 min (Figure $5 \mathrm{C}$ and $\mathrm{D}$ ) are consistent with enhanced tumour delivery, since 728 signal intensity in the area of the tumour was greater following application of US compared to omission of US. Signal was noted immediately beyond the tumour, indicating that the area of US exposure was potentially more extensive than necessary. However, the clearly enhanced uptake observed in Figure $5 \mathrm{~A}$ and $\mathrm{C}$

732 versus $\mathrm{D}$, does suggest that specific triggered release/delivery of a radiopharmaceutical from a liposomal carrier can be achieved in vivo. These results are in accordance with a previous report of delivery of a PET integrin tracer incorporated within a microbubble where focused US doubled tumour accumulation within $15 \mathrm{~min}$ in a human glioblastoma xenograft[56]. This result is likely a combination

737 of liposome opening[14], enhanced delivery[36] and potential opening of the 738 endothelial barrier[46], however which mechanism is dominant requires further investigation. 
741 Notably, it is apparent that $\left[{ }^{111} \mathrm{In}\right]$ In-HEGF-Lip can also achieve similar ( 2 \%ID/g)

742 tumour accumulation via simple passive means after $24 \mathrm{~h}$ (Figure 4E). In previous

743 studies US has been shown to enhance the delivery of PLGA nanoparticles carrying

744 microRNA miR-122 by $4-14$ fold in human colon cancer xenografts in mice[57]. The

745 reason for the lower increase reported here relative to this previous work is likely due

746 to the poor vascularity of the tumour model and lack of penetration of MBs and

$747 \quad\left[{ }^{111} \operatorname{In}\right]$ In-HEGF-Lip to the tumour core. In Figure 5E and $\mathrm{F}$ autoradiography of the

748 tumour sections show that although US-mediated cavitation increased the amount of

749 liposomes deposited in the treated peripheral 'ring' of the tumour, the core has a low

750 signal intensity regardless of the addition of US, indicating poor delivery beyond this

751 perfused ring. There was no statistical difference in the penetration depth of liposomes

752 with and without US. Contrast enhanced imaging also showed a low acoustic signal

753 intensity within the tumour core (Figure 5G). The vascularity of the tumours was further

754 examined via MRI and contrast enhanced US and compared with other tumour models

755 (Supplementary Figure 11). The results showed that the MDA-MB-468 tumours are

756 very poorly vascularised relative to a CT26 tumour model, which has been previously

757 used for US enhanced delivery[58]. However, the CT26 tumour cell line is of murine

758 origin and does not overexpress EGFR, making it an unsuitable model for our studies.

759

760 If the MBs cannot enter the tumour environment then it will not be possible for them to

761 cavitate and enhance delivery and release of the $\left[{ }^{111} \ln \right] \ln -H E G F-L i p$. However, it is

762 notable that even in a poorly vascularised tumour, MDA-MB-468, the application of

763 US had a statistically significant impact on delivery, which might indicate therapeutic

764 potential even for poorly vascularised tumours and requires further investigation.

765 However, this outcome provides an important point to examine the vasculature of the 
766

767

768

769

770

771

772

773

774

\section{Conclusion}

776 This exploratory research has shown that radiopharmaceutical loaded nanoparticles

778

779

780

781

782

783

784

785

786 Limitations

787 As this research combined existing technologies minimal characterisation was 788 789 790

tumour line chosen in conjunction with a molecular target for enhancing delivery via US. Whether using this US approach to achieve rapid delivery of high peak intratumour concentration is therapeutically preferable to the slow prolonged delivery achieved by the EPR effect also requires further investigation. It is also possible cavitation within the tumour could also have an impact upon the vasculature causing potential changes and impacting repeat treatments. As such investigating tumour vasculature before and after treatment would be recommended in the future. combined with cavitation enhanced delivery does have clinical potential. Each stage requires optimisation and further investigation but the current strategy is relevant for radiopharmacies, only impacts cells overexpressing EGFR, has extended the circulation time, changed the biodistribution and increased tumour uptake warranting further study. This research also highlights potential pitfalls such as the impact of vasculature on US enhanced delivery which is another key variable for tumour target selection along with target receptor/antigen presentation. performed for the nanoparticles and the radiotherapeutic which have been examined in previous publications. Further characterisation/optimisation of the loading techniques and the nanoparticles will be required before progressing to other 
experiments. Notably optimising radiolabelling to remove the SE centrifugation, which

792

793

794

795

796

797

798

799

800

801

802

803

804

805

806

807

808

809

810

811

812

813

814

815

816

817

818

819

820

821

822

823

will not be desirable in a radiopharmacy. Use of physical stimuli to deliver therapeutics must overcome physical barriers in the human body. Xenograft tumours in a mouse model are likely to be different morphologically from human tumours and certainly in terms of access for US. Development of ex vivo models replicating physical conditions found within the human body may be a means to more accurately assess US enhanced delivery of radiopharmaceuticals for a clinical setting. Further improvements in cavitation assisted delivery may potentially be realised through optimisation of US exposure conditions paired with model-specific perfusion analysis. Improved matching of the pharmacokinetics of the liposomes with agents used to seed cavitation may help sustain and improve the delivery over longer periods, i.e. the replacement of MBs with polymeric nanocups described by Myers et al[59], a formulation which nucleates cavitation over an extended period.

\section{References}

[1] M.R. Gill, N. Falzone, Y. Du, K.A. Vallis, Targeted radionuclide therapy in combinedmodality regimens, The Lancet Oncology, 18 (2017) e414-e423.

[2] J. Strosberg, G. El-Haddad, E. Wolin, A. Hendifar, J. Yao, B. Chasen, E. Mittra, P.L. Kunz, M.H. Kulke, H. Jacene, D. Bushnell, T.M. O’Dorisio, R.P. Baum, H.R. Kulkarni, M. Caplin, R. Lebtahi, T. Hobday, E. Delpassand, E. Van Cutsem, A. Benson, R. Srirajaskanthan, M. Pavel, J. Mora, J. Berlin, E. Grande, N. Reed, E. Seregni, K. Öberg, M. Lopera Sierra, P. Santoro, T. Thevenet, J.L. Erion, P. Ruszniewski, D. Kwekkeboom, E. Krenning, Phase 3 Trial of 177LuDotatate for Midgut Neuroendocrine Tumors, New England Journal of Medicine, 376 (2017) 125-135.

[3] M. Boegemann, A.J. Schrader, K. Rahbar, 177Lu-PSMA-Therapie, Der Urologe, 56 (2017) 1440-1444.

[4] B. Cornelissen, K.A. Vallis, Targeting the nucleus: an overview of Auger-electron radionuclide therapy, Current drug discovery technologies, 7 (2010).

[5] J.V. Leyton, M.D. Hu, C. Gao, P.V. Turner, J.E. Dick, M. Minden, R.M. Reilly, Auger Electron Radioimmunotherapeutic Agent Specific for the CD123(+)/CD131(-) Phenotype of the Leukemia Stem Cell Population, Journal of Nuclear Medicine, 52 (2011) 1465-1473. 

pharmacokinetic, biodistribution, toxicology, and dosimetry studies of In-111-DTPA-human epidermal growth factor: An Auger electron-emitting radiotherapeutic agent for epidermal growth factor receptor-positive breast cancer, Journal of Nuclear Medicine, 47 (2006) 10231031.

[7] P. Lai, W. Daear, R. Löbenberg, E.J. Prenner, Overview of the preparation of organic polymeric nanoparticles for drug delivery based on gelatine, chitosan, poly(d,I-lactide-coglycolic acid) and polyalkylcyanoacrylate, Colloids and Surfaces B: Biointerfaces, 118 (2014) 154-163. [8] A.Z. Wang, K. Yuet, L.F. Zhang, F.X. Gu, M. Huynh-Le, A.F. Radovic-Moreno, P.W. Kantoff, N.H. Bander, R. Langer, O.C. Farokhzad, ChemoRad nanopartides: a novel multifunctional nanoparticle platform for targeted delivery of concurrent chemoradiation, Nanomedicine, 5 (2010) 361-368.

[9] E. Vegt, M. Melis, A. Eek, M. de Visser, M. Brom, W.J.G. Oyen, M. Gotthardt, M. de Jong, O.C. Boerman, Renal uptake of different radiolabelled peptides is mediated by megalin: SPECT and biodistribution studies in megalin-deficient mice, European journal of nuclear medicine and molecular imaging, 38 (2011) 623-632.

[10] L. Song, N. Falzone, K.A. Vallis, EGF-coated gold nanoparticles provide an efficient nanoscale delivery system for the molecular radiotherapy of EGFR-positive cancer, International Journal of Radiation Biology, 92 (2016) 716-723. [11] K.A. Vallis, R.M. Reilly, D. Scollard, P. Merante, A. Brade, S. Velauthapillai, C. Caldwell, I. Chan, M. Freeman, G. Lockwood, N.A. Miller, B. Cornelissen, J. Petronis, K. Sabate, Phase I trial to evaluate the tumor and normal tissue uptake, radiation dosimetry and safety of (111)In-DTPA-human epidermal growth factor in patients with metastatic EGFR-positive breast cancer, American journal of nuclear medicine and molecular imaging, 4 (2014) 181192.

850 [12] G. Milano, J.P. Spano, B. Leyland-Jones, EGFR-targeting drugs in combination with cytotoxic agents: From bench to bedside, a contrasted reality, British Journal of Cancer, 99 (2008) 1-5. active tumor targeting of nanocarriers for anti-cancer drug delivery, Journal of Controlled Release, 148 (2010) 135-146. [14] S.M. Graham, R. Carlisle, J.J. Choi, M. Stevenson, A.R. Shah, R.S. Myers, K. Fisher, M.-B. Peregrino, L. Seymour, C.C. Coussios, Inertial cavitation to non-invasively trigger and monitor intratumoral release of drug from intravenously delivered liposomes, Journal of Controlled Release, 178 (2014) 101-107.

860 [15] S.D. Li, L. Huang, Pharmacokinetics and biodistribution of nanoparticles, Molecular 861 Pharmaceutics, 5 (2008) 496-504.

862 [16] H. Maeda, J. Wu, T. Sawa, Y. Matsumura, K. Hori, Tumor vascular permeability and the 863 EPR effect in macromolecular therapeutics: A review, Journal of Controlled Release, 65 864 (2000) 271-284.

865 [17] S. Wilhelm, A.J. Tavares, Q. Dai, S. Ohta, J. Audet, H.F. Dvorak, W.C.W. Chan, Analysis of nanoparticle delivery to tumours, Nature Reviews Materials, 1 (2016) 16014.

867 [18] Y. Barenholz, Doxil ${ }^{\circledR}$ - The first FDA-approved nano-drug: Lessons learned, Journal of 868 Controlled Release, 160 (2012) 117-134.

869 [19] T.M. Allen, P.R. Cullis, Liposomal drug delivery systems: From concept to clinical applications, Advanced Drug Delivery Reviews, 65 (2013) 36-48. 
872 Lee, M.C. Woodle, D.D. Lasic, C. Redemann, F.J. Martin, Sterically stabilized liposomes: Improvements in pharmacokinetics and antitumor therapeutic efficacy, Proceedings of the National Academy of Sciences of the United States of America, 88 (1991) 11460-11464. [21] L.D. Mayer, M.B. Bally, P.R. Cullis, Uptake of adriamycin into large unilamellar vesicles in response to a $\mathrm{pH}$ gradient, Biochimica et Biophysica Acta (BBA) - Biomembranes, 857 (1986) 123-126.

[22] U. Pick, Liposomes with a large trapping capacity prepared by freezing and thawing of sonicated phospholipid mixtures, Archives of Biochemistry and Biophysics, 212 (1981) 186-

880194. [23] O.C. Boerman, G. Storm, W.J.G. Oyen, L. Van Bloois, J.W.M. Van der Meer, R.A.M.J. Claessens, D.J.A. Crommelin, F.H.M. Corstens, Sterically stabilized liposomes labeled with Indium-111 to image focal infection, Journal of Nuclear Medicine, 36 (1995) 1639-1644. [24] M.R. Lewis, R. Kannan, Development and applications of radioactive nanoparticles for imaging of biological systems, Wiley Interdisciplinary Reviews: Nanomedicine and Nanobiotechnology, 6 (2014) 628-640. [25] P.L. Beaumier, K.J. Hwang, An efficient method for loading Indium-111 into liposomes using acetylacetone, Journal of Nuclear Medicine, 23 (1982) 810-815. [26] K.J. Hwang, J.E. Merriam, P.L. Beaumier, K.F.S. Luk, Encapsulation, with high efficiency, of radioactive metal ions in liposomes, BBA - General Subjects, 716 (1982) 101-109. [27] L.D.G. Mota, A.L.B. De Barros, L.L. Fuscaldi, M.C. De Oliveira, V.N. Cardoso, Longcirculating and $\mathrm{pH}$-sensitive liposome preparation trapping a radiotracer for inflammation site detection, Journal of Nanoscience and Nanotechnology, 15 (2015) 4149-4158. [28] B. Goins, R. Klipper, A.S. Rudolph, R.O. Cliff, R. Blumhardt, W.T. Phillips, Biodistribution and imaging studies of technetium-99m-labeled liposomes in rats with focal infection, Journal of Nuclear Medicine, 34 (1993) 2160-2168. [29] T.H. Chow, Y.Y. Lin, J.J. Hwang, H.E. Wang, Y.L. Tseng, V.F. Pang, S.J. Wang, J. WhangPeng, G. Ting, Diagnostic and therapeutic evaluation of 111 In-vinorelbine-liposomes in a human colorectal carcinoma HT-29/luc-bearing animal model, Nuclear Medicine and Biology, 35 (2008) 623-634. [30] D.S. Ferreira, F.A. Boratto, V.N. Cardoso, R. Serakides, S.O. Fernandes, L.A.M. Ferreira, M.C. Oliveira, Alendronate-coated long-circulating liposomes containing 99mtechnetiumceftizoxime used to identify osteomyelitis, International Journal of Nanomedicine, 10 (2015) 2441-2450.

[31] A.I. Jensen, G.W. Severin, A.E. Hansen, F.P. Fliedner, R. Eliasen, L. Parhamifar, A. Kjær, T.L. Andresen, J.R. Henriksen, Remote-loading of liposomes with manganese-52 and in vivo evaluation of the stabilities of 52Mn-DOTA and 64CU-DOTA using radiolabelled liposomes and PET imaging, Journal of Controlled Release, 269 (2018) 100-109.

[32] J.R. Henriksen, A.L. Petersen, A.E. Hansen, C.G. Frankær, P. Harris, D.R. Elema, A.T. Kristensen, A. Kjær, T.L. Andresen, Remote Loading of 64Cu2+ into Liposomes without the Use of Ion Transport Enhancers, ACS Applied Materials \& Interfaces, 7 (2015) 22796-22806. [33] M. Rotman, M.M. Welling, A. Bunschoten, M.E. de Backer, J. Rip, R.J.A. Nabuurs, P.J. Gaillard, M.A. van Buchem, S.M. van der Maarel, L. van der Weerd, Enhanced glutathione PEGylated liposomal brain delivery of an anti-amyloid single domain antibody fragment in a mouse model for Alzheimer's disease, J Control Release, 203 (2015) 40-50. 

Bioconjugate Chem., 18 (2007) 2061-2067. [36] R. Carlisle, J. Choi, M. Bazan-Peregrino, R. Laga, V. Subr, L. Kostka, K. Ulbrich, C.C. Coussios, L.W. Seymour, Enhanced tumor uptake and penetration of virotherapy using polymer stealthing and focused ultrasound, Journal of the National Cancer Institute, 105 (2013) 1701-1710. [37] R. Myers, M. Grundy, C. Rowe, C.M. Coviello, L. Bau, P. Erbs, J. Foloppe, J.-M. Balloul, C. Story, C.C. Coussios, R. Carlisle, Ultrasound-mediated cavitation does not decrease the activity of small molecule, antibody or viral-based medicines, International Journal of Nanomedicine, 13 (2018) 337-349. [38] R.M. Reilly, J. Gariépy, Factors Influencing the Sensitivity of Tumor Imaging with a Receptor-Binding Radiopharmaceutical, Journal of Nuclear Medicine, 39 (1998) 1036-1043. [39] M. Maji, S. Mazumder, S. Bhattacharya, S.T. Choudhury, A. Sabur, M. Shadab, P. Bhattacharya, N. Ali, A Lipid Based Antigen Delivery System Efficiently Facilitates MHC ClassI Antigen Presentation in Dendritic Cells to Stimulate CD8(+) T Cells, Scientific reports, 6 (2016) 27206-27206.

934

935 [40] J.-P. Colletier, B. Chaize, M. Winterhalter, D. Fournier, Protein encapsulation in liposomes: efficiency depends on interactions between protein and phospholipid bilayer, BMC Biotechnology, 2 (2002) 9-9. [41] A. Akbarzadeh, R. Rezaei-Sadabady, S. Davaran, S.W. Joo, N. Zarghami, Y. Hanifehpour, M. Samiei, M. Kouhi, K. Nejati-Koshki, Liposome: classification, preparation, and applications, Nanoscale Research Letters, 8 (2013) 102-102.

[42] J.C. Giddings, F.J. Yang, M.N. Myers, Flow field-flow fractionation as a methodology for protein separation and characterization, Analytical Biochemistry, 81 (1977) 395-407.

942

943 [43] R.E. Weiner, Role of Phosphate-Containing Compounds in the Transfer of Indium-111 and Gallium-67 from Transferrin to Ferritin, Journal of Nuclear Medicine, 30 (1989) 70-79. [44] I. Lentacker, B. Geers, J. Demeester, S.C. De Smedt, N.N. Sanders, Design and evaluation of doxorubicin-containing microbubbles for ultrasound-triggered doxorubicin delivery: Cytotoxicity and mechanisms involved, Molecular Therapy, 18 (2010) 101-108. [45] B. Geers, I. Lentacker, N.N. Sanders, J. Demeester, S. Meairs, S.C. De Smedt, Selfassembled liposome-loaded microbubbles: The missing link for safe and efficient ultrasound triggered drug-delivery, Journal of Controlled Release, 152 (2011) 249-256.

950

951 [46] A. Bouakaz, A. Zeghimi, A.A. Doinikov, Sonoporation: Concept and mechanisms, in: Advances in Experimental Medicine and Biology, 2016, pp. 175-189.

952 [47] C. Mannaris, L. Bau, M. Grundy, M. Gray, H. Lea-Banks, A. Seth, B. Teo, R. Carlisle, E. 953 Stride, C.C. Coussios, Microbubbles, Nanodroplets and Gas-Stabilizing Solid Particles for Ultrasound-Mediated Extravasation of Unencapsulated Drugs: An Exposure Parameter 955 Optimization Study, Ultrasound Med. Biol., 45 (2019) 954-967.

956 [48] Y. Hu, J.M.F. Wan, A.C.H. Yu, Membrane Perforation and Recovery Dynamics in 957 Microbubble-Mediated Sonoporation, Ultrasound Med. Biol., 39 (2013) 2393-2405. 958 [49] I. Lentacker, S.C. De Smedt, J. Demeester, V. Van Marck, M. Bracke, N.N. Sanders, 959 Lipoplex-loaded microbubbles for gene delivery: A trojan horse controlled by ultrasound, 960 Advanced Functional Materials, 17 (2007) 1910-1916. 
[50] I. Lentacker, I. De Cock, R. Deckers, S.C. De Smedt, C.T.W. Moonen, Understanding ultrasound induced sonoporation: Definitions and underlying mechanisms, Advanced Drug Delivery Reviews, 72 (2014) 49-64.

[51] E. Stride, C. Porter, A.G. Prieto, Q. Pankhurst, Enhancement of Microbubble Mediated Gene Delivery by Simultaneous Exposure to Ultrasonic and Magnetic Fields, Ultrasound in Medicine \& Biology, 35 (2009) 861-868.

[52] R.M. Reilly, R. Kiarash, R.G. Cameron, N. Porlier, J. Sandhu, R.P. Hill, K. Vallis, A. Hendler, J. Gariépy, 111In-Labeled EGF Is Selectively Radiotoxic to Human Breast Cancer Cells Overexpressing EGFR, Journal of Nuclear Medicine, 41 (2000) 429-438.

[53] A. Gabizon, H. Shmeeda, Y. Barenholz, Pharmacokinetics of Pegylated Liposomal Doxorubicin, Clinical Pharmacokinetics, 42 (2003) 419-436.

[54] E. Thomas, J.U. Menon, J. Owen, I. Skaripa-Koukelli, S. Wallington, M. Gray, C. Mannaris, V. Kersemans, D. Allen, P. Kinchesh, S. Smart, R. Carlisle, K.A. Vallis, Ultrasoundmediated cavitation enhances the delivery of an EGFR-targeting liposomal formulation designed for chemo-radionuclide therapy, Theranostics, 9 (2019) 5595-5609. [55] C.D. Arvanitis, M. Bazan-Peregrino, B. Rifai, L.W. Seymour, C.C. Coussios, CavitationEnhanced Extravasation for Drug Delivery, Ultrasound Med. Biol., 37 (2011) 1838-1852. [56] Y.-H. Chung, P.-H. Hsu, C.-W. Huang, W.-C. Hsieh, F.-T. Huang, W.-C. Chang, H. Chiu, S.T. Hsu, T.-C. Yen, Evaluation of Prognostic Integrin $\alpha 2 \beta 1$ PET Tracer and Concurrent Targeting Delivery Using Focused Ultrasound for Brain Glioma Detection, Molecular Pharmaceutics, 11 (2014) 3904-3914.

[57] T.Y. Wang, J.W. Choe, K. Pu, R. Devulapally, S. Bachawal, S. Machtaler, S.M. Chowdhury, R. Luong, L. Tian, B. Khuri-Yakub, J. Rao, R. Paulmurugan, J.K. Willmann, Ultrasound-guided delivery of microRNA loaded nanoparticles into cancer, Journal of Controlled Release, 203 (2015) 99-108.

[58] J.J. Kwan, R. Myers, C.M. Coviello, S.M. Graham, A.R. Shah, E. Stride, R.C. Carlisle, C.C. Coussios, Ultrasound-Propelled Nanocups for Drug Delivery, Small (Weinheim an der Bergstrasse, Germany), 11 (2015) 5305-5314.

[59] R. Myers, C. Coviello, P. Erbs, J. Foloppe, C. Rowe, J. Kwan, C. Crake, S. Finn, E. Jackson, J.-M. Balloul, C. Story, C. Coussios, R. Carlisle, Polymeric Cups for Cavitation-mediated Delivery of Oncolytic Vaccinia Virus, Molecular Therapy, 24 (2016) 1627-1633. 
1008 